University of Nebraska - Lincoln

DigitalCommons@University of Nebraska - Lincoln

December 1990

\title{
Magnetism of rare-earth-transition-metal nanoscale multilayers. I. Experiments on Dy/Co, Dy/Fe, and Tb/Fe
}

\author{
Z.S. Shan \\ University of Nebraska - Lincoln \\ David J. Sellmyer \\ University of Nebraska-Lincoln, dsellmyer@unl.edu
}

Follow this and additional works at: https://digitalcommons.unl.edu/physicssellmyer

Part of the Physics Commons

Shan, Z.S. and Sellmyer, David J., "Magnetism of rare-earth-transition-metal nanoscale multilayers. I. Experiments on Dy/Co, Dy/Fe, and Tb/Fe" (1990). David Sellmyer Publications. 122.

https://digitalcommons.unl.edu/physicssellmyer/122

This Article is brought to you for free and open access by the Research Papers in Physics and Astronomy at DigitalCommons@University of Nebraska - Lincoln. It has been accepted for inclusion in David Sellmyer Publications by an authorized administrator of DigitalCommons@University of Nebraska - Lincoln. 


\title{
Magnetism of rare-earth - transition-metal nanoscale multilayers. I. Experiments on $\mathrm{Dy} / \mathrm{Co}, \mathrm{Dy} / \mathrm{Fe}$, and $\mathrm{Tb} / \mathrm{Fe}$
}

\author{
Z. S. Shan* and D. J. Sellmyer \\ Behlen Laboratory of Physics and Center for Materials Research and Analysis, \\ University of Nebraska-Lincoln, Lincoln, Nebraska 68588-0111 \\ (Received 16 January 1990; revised manuscript received 30 April 1990)
}

\begin{abstract}
Experimental investigations of magnetic and structural properties for rare-earth-transition-metal (RE-TM) compositionally modulated films (CMF) are presented in this paper. In particular, $A^{\mathrm{RE}} / B^{\mathrm{TM}}$ including $A^{\mathrm{RE}}=\mathrm{Dy}$ and $\mathrm{Tb}$ and $B^{\mathrm{TM}}=\mathrm{Fe}, \mathrm{Co}$, and $\mathrm{Ni}$, are studied. The layer thickness and temperature dependence of magnetic properties, which can be interpreted in terms of the antiferromagnetic coupling of RE and TM moments and their atomic distributions, are reported. These $A^{\mathrm{RE}} / B^{\mathrm{TM}} \mathrm{CMF}$ with nanoscale layer thicknesses exhibit perpendicular magnetic anisotropy (PMA) and the range of the layer thickness required for PMA is determined. The origin of PMA is also discussed.
\end{abstract}

\section{INTRODUCTION}

Rare-earth-transition-metal thin films have been of great interest and their properties have been studied intensively. The reasons for this interest include both interface and quasi-two-dimensional magnetism and also their promising applications as magnetic information storage media used, for example, perpendicular and magnetooptical recording. Among the RE-TM homogeneous films, Gd-Co and Gd-Fe were the first found to possess perpendicular anisotropy. ${ }^{1}$ Subsequently, a number of $A^{\mathrm{RE}}-B^{\mathrm{TM}}\left(A^{\mathrm{RE}}=\mathrm{Nd}, \mathrm{Tb}, \mathrm{Gd}\right.$, etc.; $\left.B^{\mathrm{TM}}=\mathrm{Co}, \mathrm{Fe}\right)$ films were reported to have perpendicular magnetic anisotropy. ${ }^{2-18}$ The origins of the perpendicular magnetic anisotropy were attributed to atomic ordering, ${ }^{19}$ anisotropic local coordination, ${ }^{20}$ shaped voids, ${ }^{21}$ strain, ${ }^{22,23}$ exchange, ${ }^{24}$ dipolar interaction, ${ }^{25,26}$ and single-ion anisotropy. ${ }^{27-32}$ Among these possible origins, mostly it has been argued that the dipolar interactions and single-ion anisotropy are the main sources of perpendicular magnetic anisotropy (PMA). It is noticed that the anisotropic short-range pair correlations of atoms play an important role for both dipolar interaction and single-ion models.

In recent years, compositionally modulated films $(\mathrm{CMF})$ or multilayers have enjoyed more and more attention for their attractive properties, and a number of CMF have been studied. Some examples are $A^{\mathrm{RE}} / B^{\mathrm{TM}}$ multilayers $\left(A^{\mathrm{RE}}=\mathrm{Tb}, \mathrm{Dy}\right.$, and $\mathrm{Nd}$, and $\left.B^{\mathrm{TM}}=\mathrm{Co}, \mathrm{Fe}\right){ }^{33-46}$ $\mathrm{Pd} / \mathrm{Co},{ }^{47-54} \mathrm{Au} / \mathrm{Co},{ }^{55}$ and $\mathrm{Fe} / \mathrm{Ag},{ }^{56}$ and the results suggest that the CMF constitutes a fertile area of pure and applied science. In particular, if one imagines making an $A / B$ multilayer with layer thicknesses $d_{A}$ and $d_{B}$, then as $d_{A}$ and $d_{B}$ decrease to three or two atomic diameters, clearly there will be anisotropic pair correlations with an excess of $A A$ and $B B$ atom pairs in the film plane and $A B$ atom pairs perpendicular to the film plane. This then suggested an important method which offers an extra degree of freedom to control the anisotropic pair distributions of $A$ and $B$ atoms and consequently to influence the
PMA and other magnetic properties.

The $A^{\mathrm{RE}} / B^{\mathrm{TM}} \mathrm{CMF}\left(A^{\mathrm{RE}}=\right.$ the heavy RE, Dy and $\mathrm{Tb}$ and $B^{\mathrm{TM}}=\mathrm{Co}, \mathrm{Fe}, \mathrm{Ni}$ ) are studied in this paper because of the following facts. (1) The heavy RE atoms have large atomic magnetic moments, and the magnetic moments of RE and TM are antiferromagnetically coupled. (2) Dy and Tb atoms have large random single-ion anisotropy. Properties (1) and (2) may lead to large anisotropy and coercivity for $A^{\mathrm{RE}} / B^{\mathrm{TM}} \mathrm{CMF}$. (3) Systematic studies on the layer thickness and temperature dependence are essential for the understanding micromagnetic structure and further controlling the magnetic properties.

In the second paper of this series we develop a theoretical model for understanding both the magnetization distribution and perpendicular magnetic anisotropy. This paper will be denoted hereafter as paper II. A third paper (III) will be devoted to other $A^{\mathrm{RE}} / B^{\mathrm{TM}} \mathrm{CMF}$ in which the RE is a light rare earth such as $\mathrm{Nd}$, or where the RE possesses small or no single-ion anisotropy (e.g., $\mathrm{Er}$ and/or Gd).

The magnetic structure of the artificially deposited CMF may be represented schematically as shown in Fig. 1. The CMF structure with the thick layers of $\mathrm{RE}$ and TM is shown in Fig. 1(a), which is composed of three regions: the inner $\mathrm{RE}$ region which is magnetically disordered at room temperature for $\mathrm{Dy}$ and $\mathrm{Tb}$; the interface region where the $R E$ and TM are antiferromagnetically coupled and their magnetic moments may be compensated somewhere; and the inner TM region which is ferromagnetically ordered. In the inner TM region the magnetic moment is usually parallel to the film plane; however in the interface region where the atomic distribution lacks symmetry, the magnetic moments may be perpendicular to the film plane, as was pointed out by Néel, ${ }^{57}$ if the anisotropy forces are large enough [this situation is not shown in Fig. 1(a)]. Therefore we come to two conclusions. (1) The CMF with very thick layer thicknesses may show characteristics of a two-phase RE and TM material because the interface contribution is negligibly 

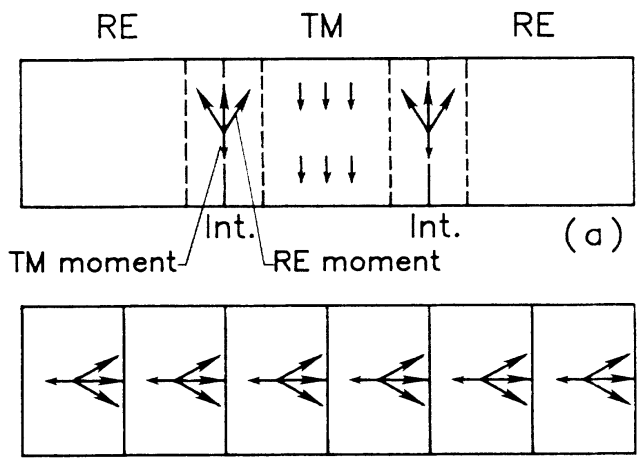

(b)

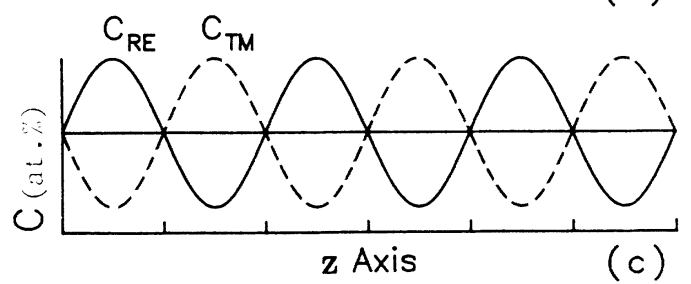

FIG. 1. The schematic representation of magnetic structure for $A^{\mathrm{RE}} / B^{\mathrm{TM}}$ as the nominal layer thickness of the heavy $\mathrm{RE}$ and TM are much thicker than the interface (a), or thinner than the nominal interface (b), and the concentration distributions of RE and TM constituents of case (b) along the film normal (c). Int. denotes interface in this figure.

small. (2) As the nominal layer thicknesses of RE and $\mathrm{TM}$ decrease until the interface dominates, then the CMF may exhibit interesting magnetic features, e.g., the net magnetic moment being perpendicular to the film plane as shown in Fig. 1(b).

In the rest of this paper the attention will be focused on the thin layer CMF where the contribution coming from the interface is dominant. The layer thickness and temperature dependence are investigated systematically for $\mathrm{Dy} / \mathrm{Co}, \mathrm{Dy} / \mathrm{Fe}$, and $\mathrm{Tb} / \mathrm{Fe} \mathrm{CMF}$, and the magnetic property of $\mathrm{Dy} / \mathrm{Ni}$ is discussed briefly as well.

The samples were grown in a multiple-gun sputtering system with the Mylar substrates for magnetic measurements and glass substrates for $\mathrm{x}$-ray diffraction. The preparation conditions and the procedure of determining the magnetic properties, such as the anisotropy, are the same as those mentioned in our previous papers. ${ }^{40,43}$ The magnetic properties were measured with a vibratingsample magnetometer at room and low temperatures. Some samples were also measured with a torquemeter for the sake of comparison. Large- and small-angle $x$-ray diffraction measurements have been performed on selected samples to determine the crystalline and compositionally modulated structure. Briefly, the structure is polycrystalline for $\mathrm{Dy} / \mathrm{Co}, \mathrm{Dy} / \mathrm{Fe}$, and $\mathrm{Tb} / \mathrm{Fe} \mathrm{CMF}$ as the individual layer thickness is greater than about $15 \AA$, and for thinner layers the structure is amorphous. Several examples will be given later for Dy/Co and $\mathrm{Dy} / \mathrm{Fe} \mathrm{CMF}$. The small- angle $\mathrm{x}$-ray diffraction patterns, as shown in Fig. 2, only show the first-order peaks for $(14 \AA \mathrm{Dy}) /(40$

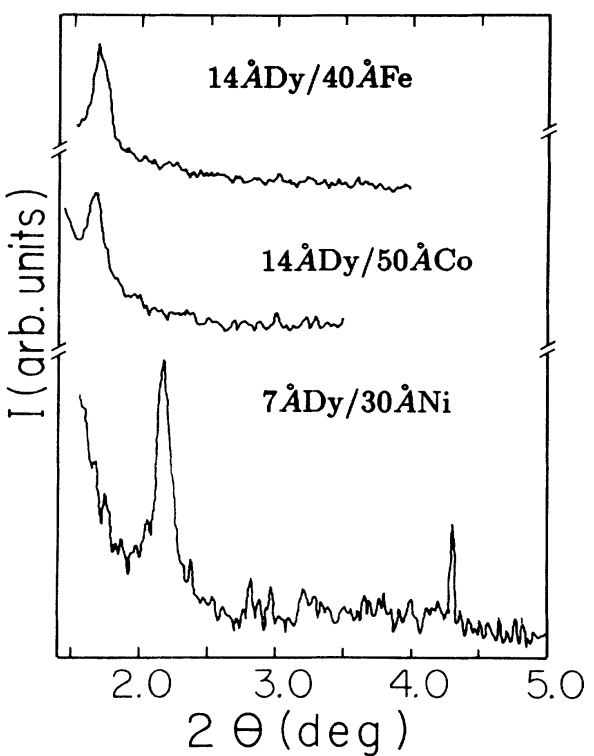

FIG. 2. Cu $K_{\alpha}$ small-angle diffraction intensity as a function of $2 \theta$ for three Dy/Co, Dy/Fe, and Dy/Ni samples.

$\AA \mathrm{Fe})$ and $(14 \AA \mathrm{Dy}) /(50 \AA \mathrm{Co})$, but also the secondorder peak for $(7 \AA \mathrm{Dy}) /(30 \AA \mathrm{Ni})$. This suggests that the compositionally modulated structure has a sinusoidal form, ${ }^{58,42}$ which is shown schematically in Fig. 1(c), for $(14 \AA \mathrm{Dy}) /(40 \AA)$ and $(14 \AA) /(50 \AA \mathrm{Co})$, and a sharper interface for $(7 \AA \mathrm{Dy}) /(30 \AA \mathrm{Ni})$.

In order to study systematically the layer thickness dependence of magnetic properties, the interval of TM layer thickness $X$ and RE layer thickness $Y$ are about one atomic diameter, namely, $2.5 \AA$ for TM and $3.5 \AA$ for RE: in some regions the intervals are only about half atomic diameter which enable us to investigate precisely the layer thickness dependence of magnetic properties.

\section{Dy-BASED CMF}

Dy/Co and Dy/Fe CMF, about 70 samples for each species, were prepared and their magnetic properties were analyzed. A few Dy/Ni CMF were also fabricated in order to compare their magnetic characteristics with those of Dy/Co and Dy/Fe CMF.

\section{A. Dy/Co CMF}

Several examples of large-angle x-ray diffraction measurements for Dy/Co CMF are shown in Fig. 3. It is seen that the structure is amorphous for individual layers thinner than $14 \AA$, while the crystalline feature, e.g., the sharper peak, becomes stronger as the layer gets thicker.

\section{Layer-thickness dependence of magnetic properties at room temperature}

The hysteresis loops of about $70 \mathrm{Dy} / \mathrm{Co}$ samples were measured at room temperature. Examples for $(5 \AA$ Dy) $/(X \AA \mathrm{Co})$ and $(3.5 \AA \mathrm{Dy}) /(X \AA \mathrm{Co})$ are shown in Fig. 


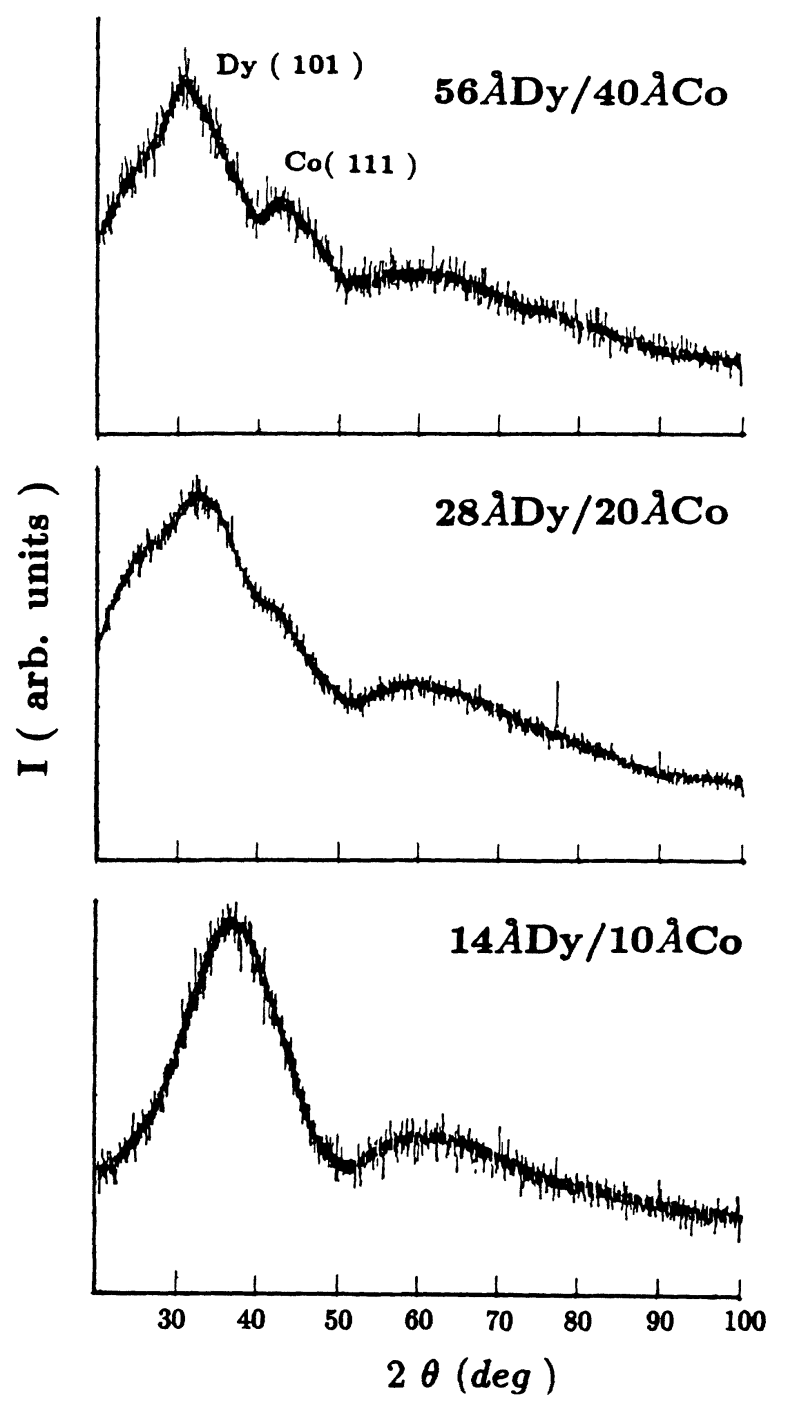

FIG. 3. $\mathrm{Cu} K_{\alpha}$ large-angle diffraction intensity as a function of $2 \theta$ for Dy/Co samples.

4. This figure shows that the magnetic anisotropy, magnetization, coercivity, and remanence change regularly as the Co and Dy layer thicknesses are changed, e.g., the coercivity $H_{c}$ is relatively small for both small and large layer thicknesses, but it is large for intermediate values. Also the films possess perpendicular anisotropy for intermediate values.

The "measured anisotropy," $K_{u}^{\prime}$, is determined by the area between the parallel and perpendicular $\sigma\left(H_{a}\right)$ curves. Figure 5 shows a plot of $\lambda K_{u}^{\prime}$ vs Co layer thickness $X$ for $(8 \AA \mathrm{Dy}) /(X \AA \mathrm{Co})$ which is a straight line for $X>15 \AA$. This behavior, which has been observed in numerous magnetic multilayers, ${ }^{41,43,47,51,55}$ can be understood in terms of the following expression:

$$
\lambda K_{u}^{\prime}=2 K_{i}+\left(K_{v}-2 \pi M_{s}^{2}\right) X,
$$

where $K_{i}, K_{v}$, and $2 \pi M_{s}^{2}$ are the interface, volume, and demagnetization anisotropy energies, respectively. The $K_{i}$ value for this series is about $0.7 \mathrm{erg} / \mathrm{cm}^{2}$. The main

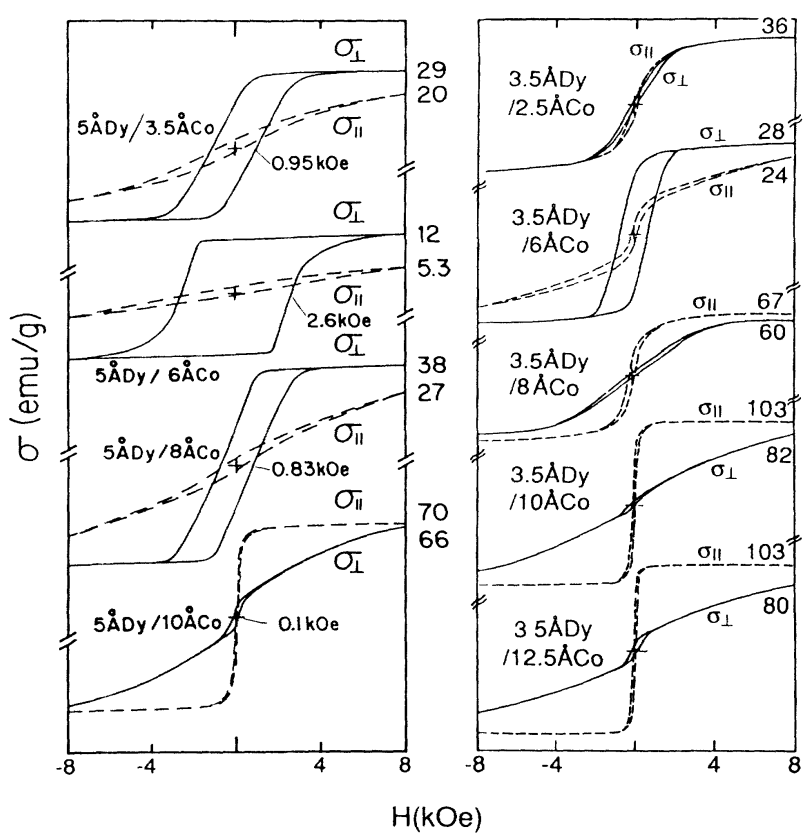

FIG. 4. Layer-thickness dependence of hysteresis loops for (5 $\AA \mathrm{Dy}) /(X \AA \mathrm{C} \mathrm{Co})$ and $(3.5 \AA \mathrm{Dy}) /(X \AA \mathrm{Co})$ at $300 \mathrm{~K}$.

point here is that Eq. (1) is no longer a straight line for $X<15 \AA$, just where the structure changes from compositionally modulated crystalline to compositionally modulated amorphous. We focus mainly on the small- $X$ and $-Y$ regions, where perpendicular anisotropy occurs.

A summary of the layer thickness dependence of the magnetization $\sigma_{\perp}$ is illustrated in Fig. 6. Based on this systematic investigation, a three dimensional diagram is shown in Fig. 7 which displays the main behavior of the

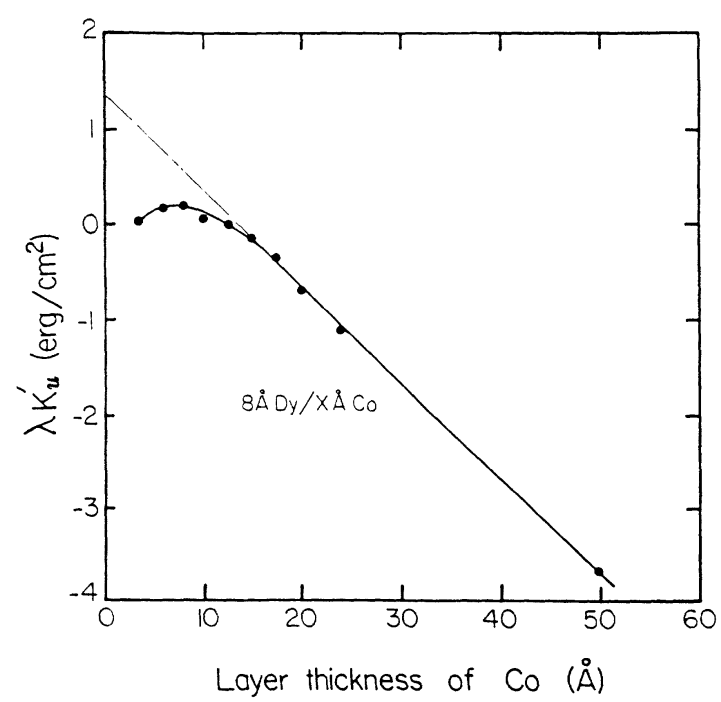

FIG. 5. The measured anisotropy $K_{u}^{\prime}$ multiplied by the bilayer thickness $\lambda$ vs the Co layer thickness for $(8 \AA \mathrm{Dy}) /(X \AA$ Co) $\mathrm{CMF}$. 


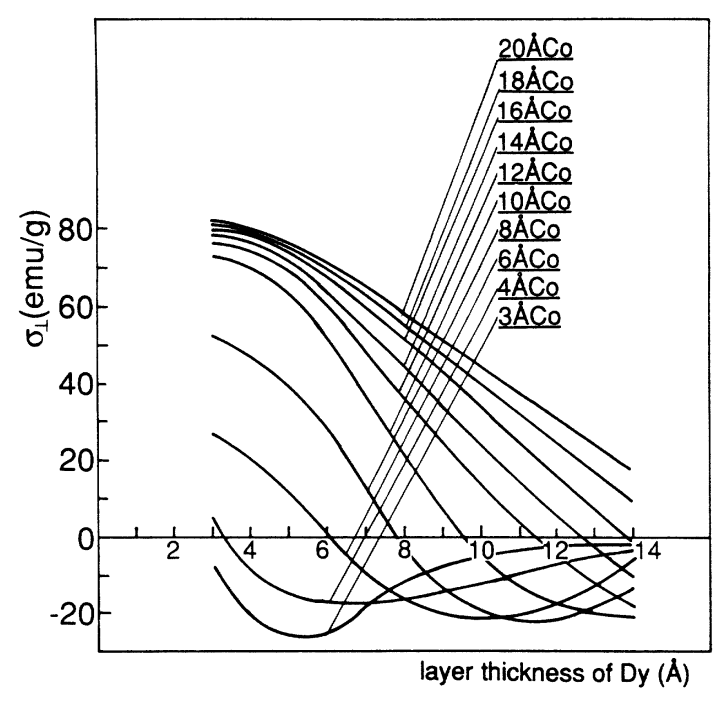

FIG. 6. Layer-thickness dependence of magnetization for ( $Y$ $\AA ̊ \mathrm{Dy}) /(X \AA \mathrm{A} \mathrm{Co}) \mathrm{CMF}$ at $H_{a}=8 \mathrm{kOe}$ and $T=300 \mathrm{~K}$. $\sigma_{\perp}$ means the magnetization was measured with the applied field perpendicular to the film plane.

magnetization. Several features should be pointed out. (1) The valley between the two peaks of magnetization, where $\left|\sigma_{\perp}\right|=0$, originates from the antiferromagnetic coupling of Co and Dy moments. Thus this valley traces the compensation points of the films of different compositions. The relationship between Dy and Co layer thicknesses for the compensation composition can be approximately expressed as $Y \simeq\left(\frac{13}{14}\right) X$. (2) The bigger peak of $\left|\sigma_{1}\right|$ on the right-hand side of the valley indicates a region where the Co magnetization dominates. The smaller hill of $\left|\sigma_{\perp}\right|$ on the left-hand side of the valley is the region where the Dy magnetization dominates. (3) As the Co and Dy layers get very thin, the magnetization $\left|\sigma_{\perp}\right|$ approaches the behavior of homogeneous Dy/Co alloys be-

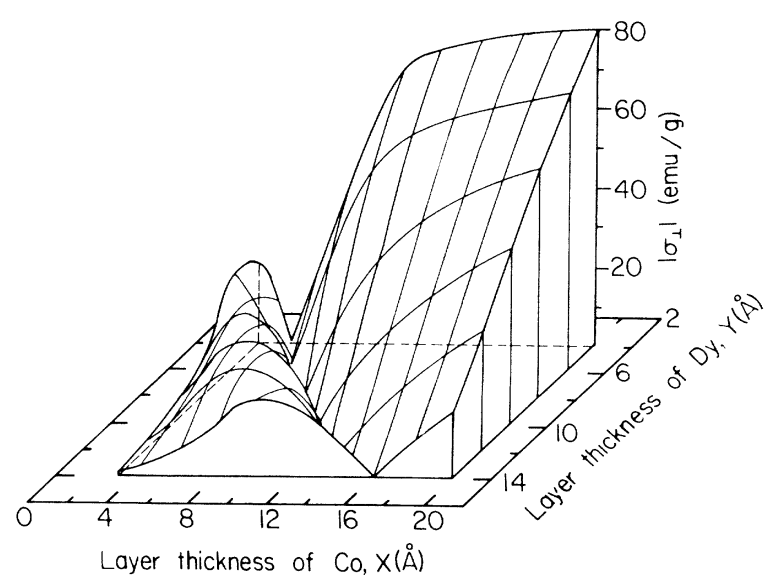

FIG. 7. The three-dimensional diagram of layer-thickness dependence of magnetization for $(Y \AA \mathrm{Dy}) /(X \AA \mathrm{Co})$ at $H_{a}=8$ $\mathrm{kOe}$, and $T=300 \mathrm{~K}$.

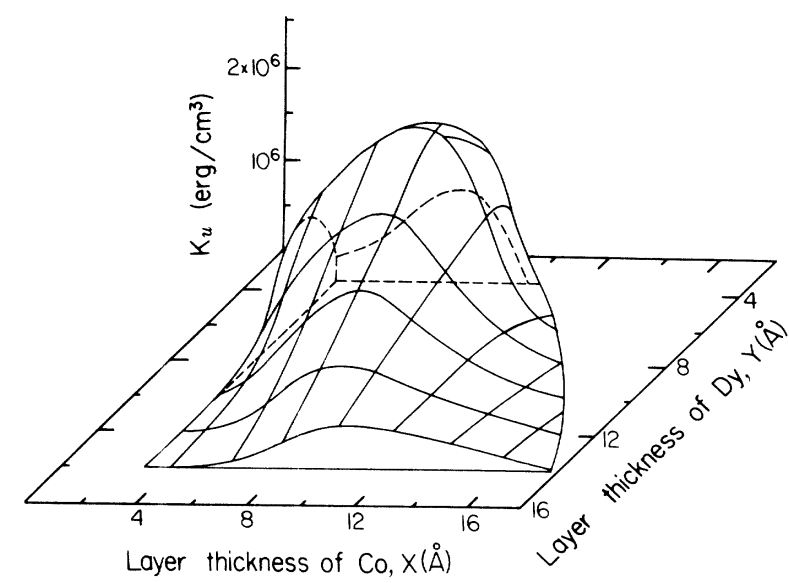

FIG. 8. The three-dimensional diagram of layer thickness dependence of intrinsic anisotropy $K_{u}$ for $(Y \AA \mathrm{Dy}) /(X \AA \mathrm{Co})$ at $300 \mathrm{~K}\left(K_{u}=K_{u}^{\prime}+2 \pi M_{s}^{2}\right)$.

cause the "layers" are so thin that an almosthomogeneous alloy is formed.

A summary of the layer thickness dependence of the intrinsic anisotropy, $K_{u}=K_{u}^{\prime}+2 \pi M_{s}^{2}$, is illustrated in Fig. 8 in three-dimensional form. There are several characteristics to be noticed. (1) The maximum value of $K_{u}$, where the sample exhibits the strongest perpendicular anisotropy, occurs at $X \sim 6 \AA, Y \sim 6 \AA$. (2) As the Co layer becomes thicker, $K_{u}$ becomes negative (not shown in this figure for clarity); therefore the easy direction of magnetization becomes parallel to the film plane. (3) The $K_{u}$ peak becomes wider and lower as the Dy layer thickness increases, and $K_{u}$ decreases rapidly and becomes negative (not shown in this figure for clarity) as the Dy layer becomes very thin, since the amorphous Co-rich alloy has in-plane anisotropy.

For technical applications, such as the magnetic recording, the reduction of the anisotropy originating from demagnetization anisotropy should be considered. Thus it is useful to determine the ranges of Co and Dy layer thicknesses exhibiting perpendicular "measured anisotropy," i.e., to determine the regions of $X$ and $Y$ where $K_{u}^{\prime}$ has positive value. This result is shown in Fig. 5 of Ref. 42. The rather broad region where $K_{u}^{\prime}>0$ suggests that Dy/Co films could be promising for magneto-optical and perpendicular recording.

A brief discussion, which may help understanding the magnetic structure of the Dy/Co CMF, is given below.

(a) Figure 8 shows that the CMF samples with intermediate layer thicknesses of $X$ and $Y$ exhibit strong perpendicular magnetic anisotropy. For example, $(6 \AA$ Dy) $/(6 \AA \mathrm{Co})$ has $\left(K_{u}\right)_{\max }$ for their layer thicknesses of Dy and Co of about 2 atomic layers, where the CMF has the maximum ratio of the "interface to volume." This means the "interfaces" of the CMF are responsible for the perpendicular magnetic anisotropy. The main feature of the interface is the anisotropic distribution of atoms there. In paper II we will discuss the origin and calculation of the perpendicular magnetic anisotropy to put this 
argument on a more clear and solid foundation.

(b) A set of $(Y \AA \mathrm{Dy}) /(X \AA \mathrm{Co})(Y / X=3.5 / 2.5$, $5.25 / 3.75,7 / 5$, and $21 / 15)$ were prepared. ${ }^{45}$ The shape of their hysteresis loops changes regularly as the nominal layer thicknesses of Dy and Co increase. We notice that all five samples have the same chemical ratio of Dy and Co. This means that the behavior is clearly controlled by the layer thickness, consequently the distributions of Dy and Co atoms, rather than the chemical composition.

(c) As pointed out above, we focus mainly on the small- $X$ and $-Y$ regions where perpendicular magnetic anisotropy occurs. But the curves of $\lambda K_{u}^{\prime}$ vs Co layer thickness in the large- $X$ and $-Y$ regions, as shown in Fig. 9, may offer more information. (1) The curve becomes a straight line for thick Co layers. But in the case of Dy/Co CMF, the intercept, and therefore the interface anisotropy $K_{i}$, increases as the Dy layer becomes thicker. This may be attributed to the interdiffusion between Co and Dy atoms. As the Dy layers become thicker, the increasing interface region due to the interdiffusion gives rise to the increase of the interface anisotropy, and the curve of $K_{l}$ vs Dy layer thickness is shown in the inset of Fig. 9. A detailed discussion of the origin of the anisotropy is given in paper II and Refs. 46 and 45 . (2) The volume anisotropy can be estimated from the slopes, i.e., $\left(K_{v}-2 \pi M_{s}^{2}\right)$, of the lines, and it is found to be $2.5 \times 10^{6}$ $\mathrm{erg} / \mathrm{cm}^{3}$ for $\left.(3.5 \AA \mathrm{Dy}) / X \AA \mathrm{Co}\right)$ and $4.9 \times 10^{6} \mathrm{erg} / \mathrm{cm}^{3}$ for $(14 \AA \mathrm{Dy}) /(X \AA \mathrm{Co})$ if the Co bulk magnetization is taken for $\boldsymbol{M}_{5}$. The volume anisotropy for pure Co is $4.5 \times 10^{6} \mathrm{erg} / \mathrm{cm}^{3}$. They are of the same order of magnitude.

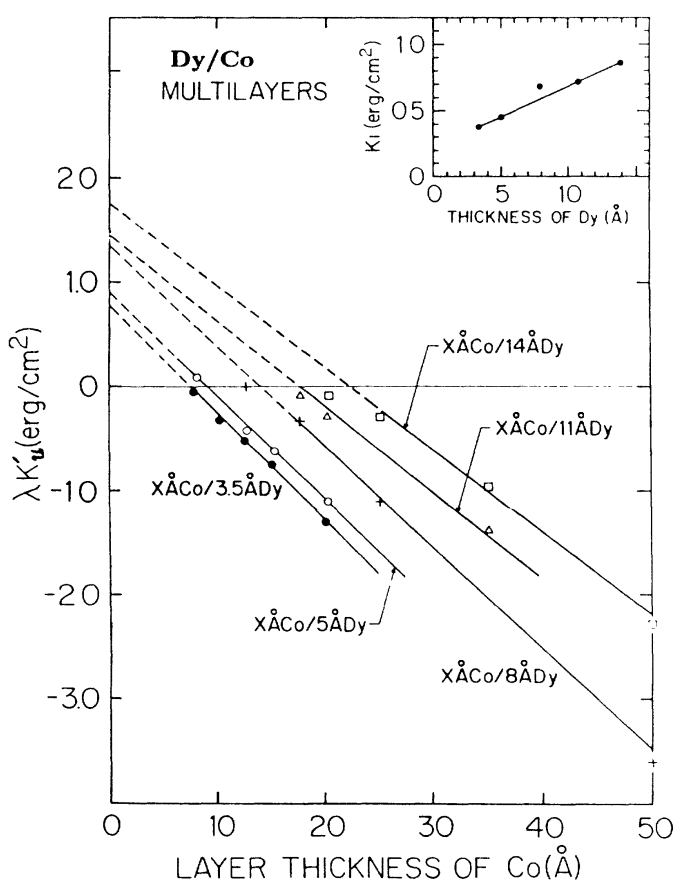

FIG. 9. The measured anisotropy $K_{u}^{\prime}$ multiplied by the bilayer thickness $\lambda$ vs the Co layer thickness for the samples with Dy layer thickness $Y=3.5,8,11$, and $14 \AA$. (d) To confirm the perpendicular anisotropy, torque measurements have been performed for $(5 \AA \mathrm{Dy}) /(X \AA$ Co) $(X=3.5,6,8,10) .{ }^{46}$ The results show that the samples possess perpendicular anisotropy for $X=3.5,6$, and 8 , and the easy axis changes from film normal to in-plane as $X$ increases from $8 \AA$ to $10 \AA$. This feature is consistent with the characteristics in Fig. 4.

\section{Temperature dependence of magnetic properties}

The temperature dependence of magnetic properties, such as magnetization, anisotropy, and coercivity, are summarized in this section. Phenomenologically, the Dy/Co CMF can be regarded as a stack of a large number of very thin Dy-Co slices with modulated composition. Therefore the character of the temperature dependence of magnetic properties for CMF structure is expected to be more complex than that of homogeneous alloys: the latter is determined only by the unique composition, and the former has the modulated distribution of composition.

Figure 10 shows the hysteresis loops $(Y \AA \mathrm{Dy}) /(6 \AA$ Co) $(Y=3.5,5,8,11$, and 14$)$ at $4.2 \mathrm{~K}$ and $300 \mathrm{~K}$. That the magnetic properties depend on temperature is seen clearly. (1) The magnetization of each sample at $4.2 \mathrm{~K}$ is larger than that at room temperature. This is due to the fact that at $4.2 \mathrm{~K}$, the Dy moment is ordered and at 300 $\mathrm{K}$ it is disordered. Especially for $(14 \AA \mathrm{Dy}) /(6 \AA \mathrm{Co})$ this feature is shown distinctly. (2) The coercivity of each sample increases remarkably as the temperature de-
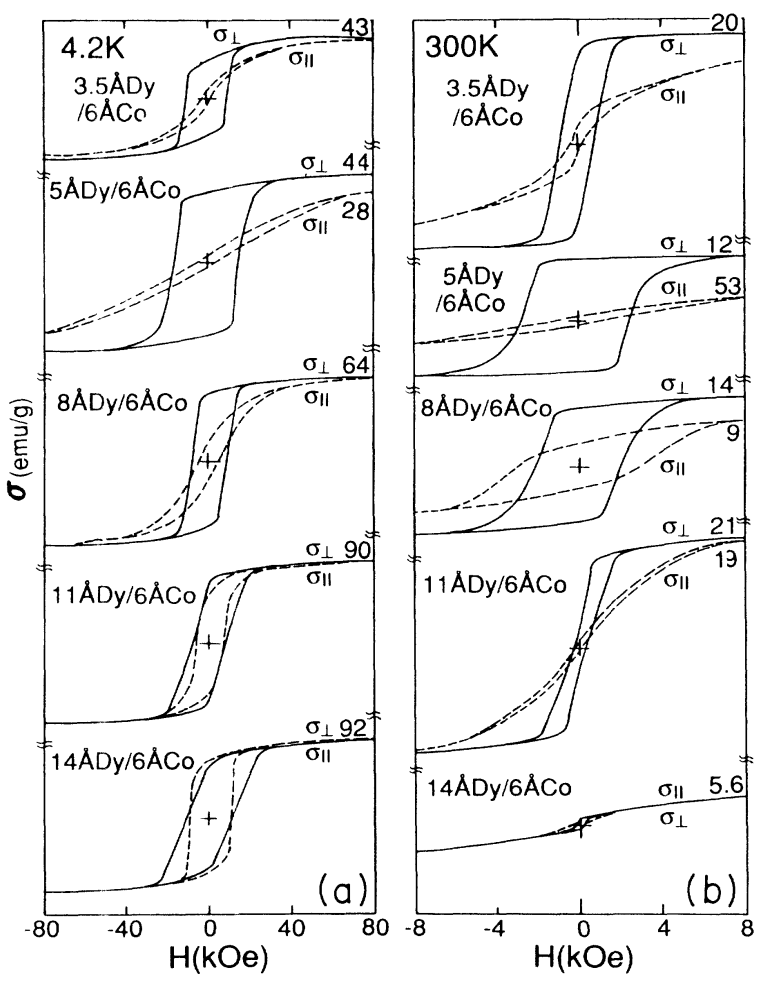

FIG. 10. Hysteresis loops for $(Y \AA \mathrm{Dy}) /(6 \AA \mathrm{Co})$ at $300 \mathrm{~K}$ and $4.2 \mathrm{~K}$. 
creases from $300 \mathrm{~K}$ to $4.2 \mathrm{~K}$ if we notice the abscissa scale of Fig. 10(a) is from $-80 \mathrm{kOe}$ to $+80 \mathrm{kOe}$, which is ten times larger than that of Fig. 10(b). (3) Both at $4.2 \mathrm{~K}$ and $300 \mathrm{~K}$, sample $(5 \AA \mathrm{Dy}) /(6 \AA \mathrm{Co})$, whose layer thicknesses of Dy and Co are about 2-atomic diameter of each species, respectively, has the maximum values of anisotropy $K_{u}$. This also implies that the interface is the main source responsible for the anisotropy. (4) At $4.2 \mathrm{~K}$, the Dy moments are ordered and the coercivity $H_{c \|}$ (the coercivity for the applied field parallel to the film plane) increases as the Dy layer becomes thicker. This implies that the Dy atomic density has an important influence on the $H_{c \|}$. Figure 11 is the summary of the Dy layer thickness dependence of magnetization and anisotropy. The features of (1)-(3) mentioned above are found easily here and even more information can be obtained. (1) Antiferromagnetic coupling between the Dy and Co magnetization is exhibited both at $300 \mathrm{~K}$ and $4.2 \mathrm{~K}$, since the magnetizations change their sign as Dy layer becomes thicker, and the compensation point moves from $Y \sim 6 \AA$ at $300 \mathrm{~K}$ to $Y \sim 4 \AA$ at $4.2 \mathrm{~K}$. (2) As the Dy layer thickness increases, the $\left|\sigma_{s}\right|$ value gets larger at $4.2 \mathrm{~K}$ because the Dy moment becomes more dominant, but the $\left|\sigma_{s}\right|$ value decreases slightly at $300 \mathrm{~K}$ since the increase of Dy layer thickness will lead to the magnetic disorder in the central part of the Dy region. (3) The maximum value of $K_{u}$ is about $1.4 \times 10^{7} \mathrm{erg} / \mathrm{cm}^{3}$ at $4.2 \mathrm{~K}$ and $2 \times 10^{6} \mathrm{erg} / \mathrm{cm}^{3}$ at $300 \mathrm{~K}$, correspondingly. The $K_{u}$ values at $4.2 \mathrm{~K}$ are much larger than those at $300 \mathrm{~K}$. This feature hints that the main origin of the anisotropy may be related to a parameter which depends on the temperature strongly, for example, the single-ion anisotropy energy of Dy, which is proportional to the Dy subnetwork magnetization
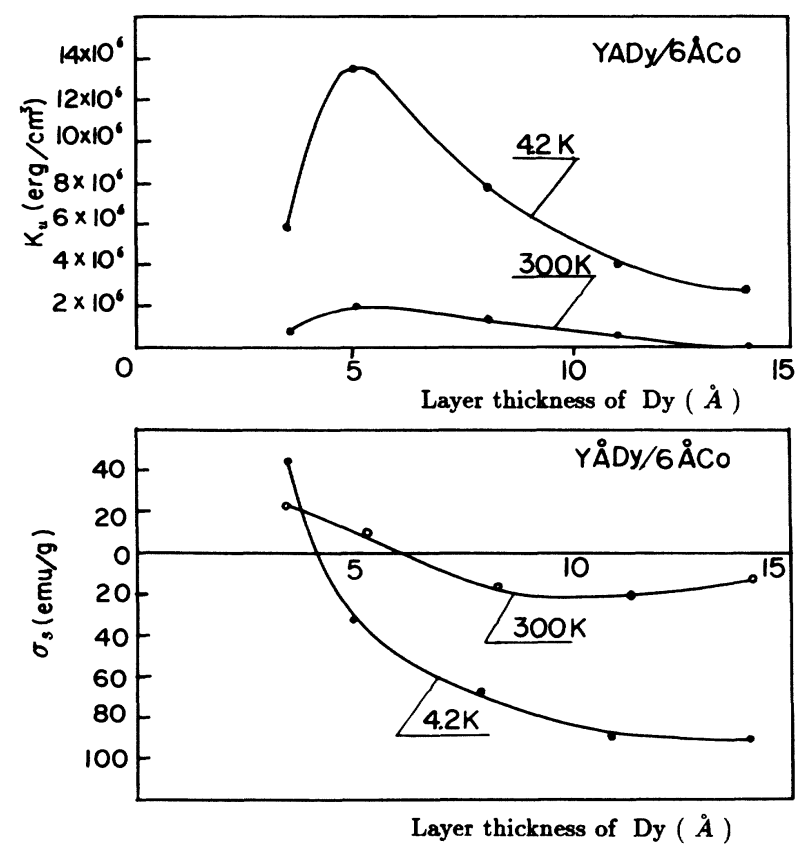

FIG. 11. Layer-thickness dependence of magnetization and anisotropy for $(Y \AA \mathrm{Dy}) /(6 \AA \mathrm{Co})$ at $300 \mathrm{~K}$ and $4.2 \mathrm{~K}$. squared. A more detailed discussion of the origin of anisotropy will be given in paper II.

An example of the temperature dependence of hysteresis loops from $4.2 \mathrm{~K}$ to $300 \mathrm{~K}$ for $(5 \AA \mathrm{Dy}) /(6 \AA \mathrm{Co})$ is shown in Fig. 12, and the temperature dependence of $\left|\sigma_{s}\right|, K_{u}$, and $H_{c}$ are shown in Fig. 13. Several characteristics should be noted. (1) The magnetization $\left|\sigma_{s}\right|$, which is dominated by Dy moment at low temperature, first decreases as temperature increases, reaches a minimum value which corresponds the compensation point at $T \simeq 190 \mathrm{~K}$, and then $\left|\sigma_{s}\right|$ increases again because the Co moment dominates. (2) The enhancement of the compensation point from about $88 \mathrm{~K}$ (Dy ordering temperature) to the present $190 \mathrm{~K}$ is due to the exchange interaction between Co and Dy atoms. This exchange interaction makes Dy subnetwork partially magnetically ordered above $88 \mathrm{~K}$. (3) The $K_{u}$ character is quite complex. For $T \leq 133 \mathrm{~K}$ and $T=300 \mathrm{~K}$, the samples exhibit perpendicular anisotropy, and for $178 \mathrm{~K}<T<200 \mathrm{~K}$ the configuration of hysteresis loops make it difficult to determine the anisotropy from the area between the parallel and perpendicular magnetization curves, approximated by the horizontal bisectors of the hysteresis loops. A rough estimation gives in-plane anisotropy without taking the contribution $2 \pi M_{s}^{2}$ into account (or very weak perpendicular anisotropy after taking the contribution of $2 \pi M_{s}^{2}$ into account) at $178 \mathrm{~K}$. The reason of this feature is not clear completely. Mostly it is owing to that the magnetic state of this sample is close to the compensation point at $T=178-200 \mathrm{~K}$ and we will point out below that the method of determining the anisotropy from the area between the parallel and perpendicular magnetization curves is unsuited in the vicinity of the compensation point. (4) The temperature dependence of $H_{c}$ displays a small peak at $T \simeq 160 \mathrm{~K}$. This character is different from the conventional behavior of homogeneous heavy rareearth-transition-metal alloys, in which the coercivity diverges near the compensation point. ${ }^{8,2}$ A possible interpretation of this discrepancy comes from the different configuration of the atomic distribution: in the CMF structure the atomic concentration of Dy and Co is compositionally modulated. Although an average of the $\mathrm{CMF}$ is in the compensation region, the individual regions are away from compensation. As a result the divergence of $H_{c}$ does not appear for $(5 \AA \mathrm{Dy}) /(6 \AA \mathrm{Co})$. To confirm this interpretation, the measurements of $H_{c}$ vs $T$

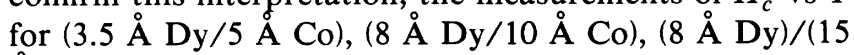
$\AA \mathrm{Co}$ ), etc., have been performed carefully and the similar results were obtained.

\section{B. Dy/Fe CMF}

There are many similarities in the magnetic characteristics between $\mathrm{Dy} / \mathrm{Co}$ and $\mathrm{Dy} / \mathrm{Fe} \mathrm{CMF}$, but some differences also will be observed in the following discussion. As in the case of Dy/Co CMF a large number ( 70) of $\mathrm{Dy} / \mathrm{Fe}$ films were made to carefully study the layer-thickness dependence of the properties. Large- and small-angle $x$-ray diffraction measurements have been performed on selected samples. Two examples of largeangle x-ray diffraction patterns are illustrated in Fig. 14, 

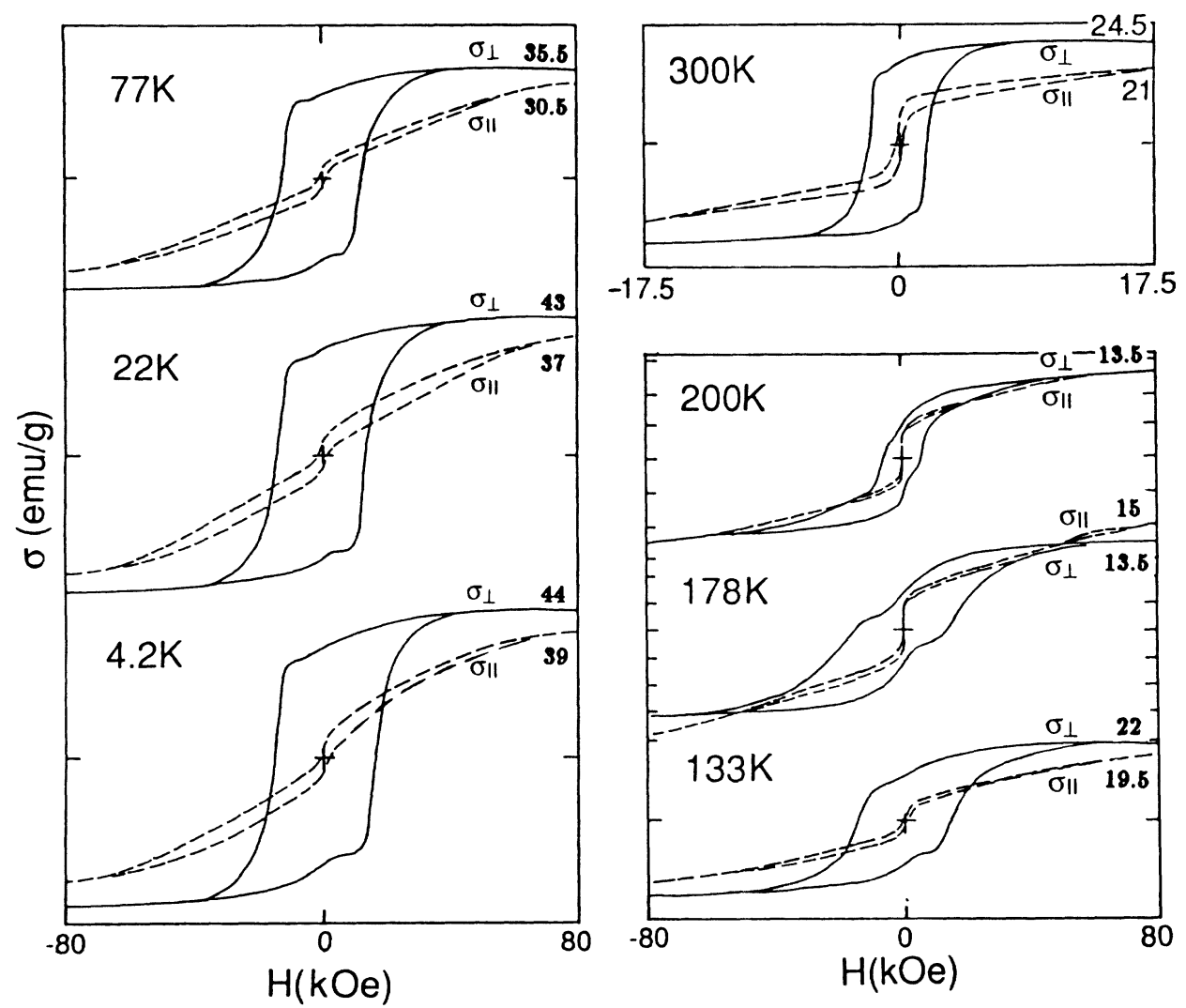

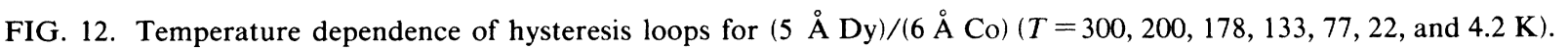

and these show a polycrystalline structure for $(56 \AA$ $\mathrm{Dy}) /(40 \AA \mathrm{Fe})$ and a noncrystalline or "nearly amorphous" pattern for $(14 \AA \mathrm{Dy}) /(15 \AA \mathrm{Fe})$. An example of small-angle pattern for $(14 \AA \mathrm{Dy}) /(40 \AA \mathrm{Fe})$ is shown in Fig. 2.

\section{The layer-thickness dependence of hysteresis loops for $(5 \AA \mathrm{Dy}) /(\mathrm{X} \AA \mathrm{Fe})$}

Figure 15 shows a detailed layer-thickness dependence of hysteresis loops for $(5 \AA \mathrm{Dy}) /(X \AA \mathrm{Ae})$ as the Fe layer

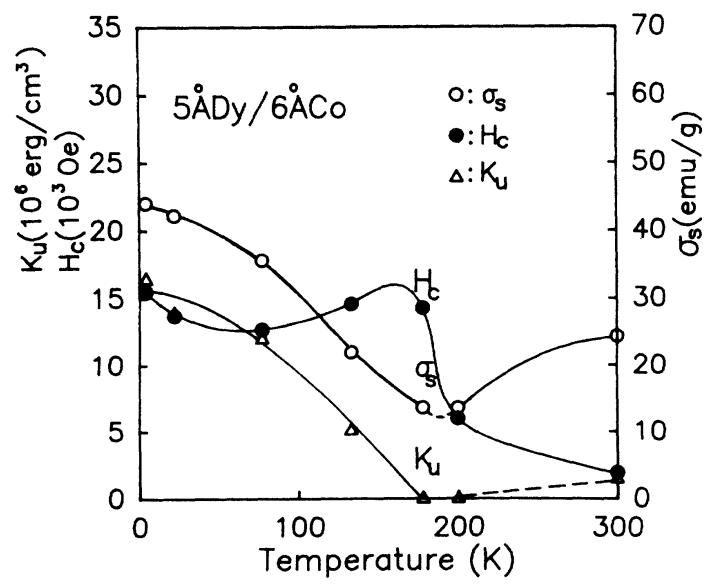

FIG. 13. Temperature dependence of saturated magnetization $\sigma_{s}$, anisotropy $K_{u}$, and coercivity $H_{c}$ for $(5 \AA \mathrm{Dy}) /(6 \AA$ $\mathrm{Co}$ ). thickness varies from $2.5 \AA$ to $40 \AA$; note especially that the interval is only $1.25 \AA$ as $X$ ranges from 2.5 to $10 \AA$. The layer-thickness dependence of magnetization and anisotropy are determined from Fig. 15 and are summarized in Fig. 16.

Several results about the magnetization can be found from Figs. 15 and 16. To understand the layer-thickness dependence of magnetization, both the antiferromagnetic coupling of Dy and Fe moments and the modulated distribution of composition have to be taken into account. (1) Sample $(5 \AA \mathrm{Dy}) /(6.25 \AA \mathrm{Fe})$ is in a state very close to the composition point. Then the Dy moment dominates as $X<6.5 \AA$ and the Fe moment dominates as $X>6.5 \AA$. (2) As $X$ increases from 2.5 to $6.5 \AA$, the magnetization magnitude of $\mathrm{Dy} / \mathrm{Fe},|\sigma|$, first increases, then decreases. This feature is due to the existence of two competitive processes: the enhancement of the Dy moments by the exchange interactions between $\mathrm{Fe}$ and $\mathrm{Dy}$ subnetwork moments as the $\mathrm{Fe}$ atomic fraction increases in this range, and the antiferromagnetic coupling between the $\mathrm{Dy}$ and $\mathrm{Fe}$ subnetwork moments. The former dominates as $X$ is close to $2.5 \AA$ and the latter prevails as $X$ approaches $6.5 \AA$. (3) As $X$ increases from 12 to $20 \AA$ the magnetization of $\sigma_{\|}$and $\sigma_{\perp}$ changes very little. This may be attributed to the fact that the pure amorphous $\mathrm{Fe}$ is disordered magnetically; ${ }^{59}$ as the $\mathrm{Fe}$ layer thickness ranges between $10 \AA$ and $20 \AA$, the Fe atomic fraction in the central region of the Fe layer is close to unity and its structure is amorphous which gives no contribution to the moments [see the similar situation for $(4.5 \AA$ 

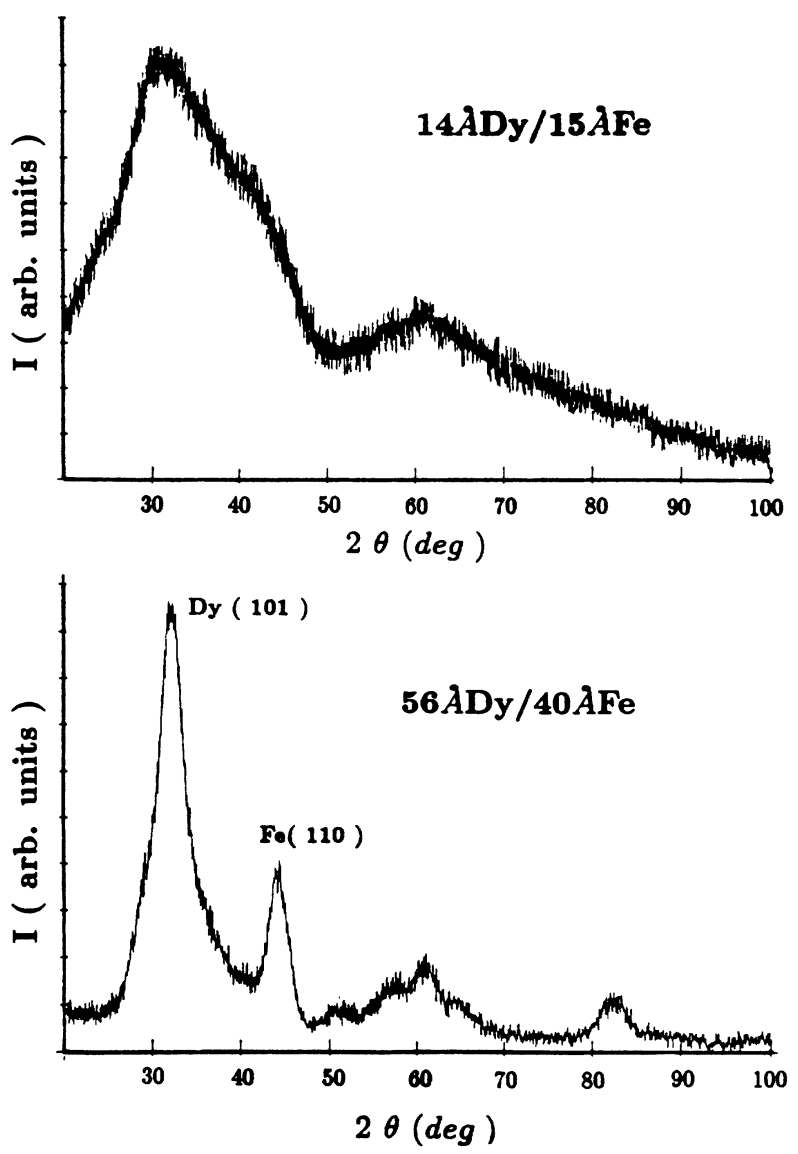

FIG. 14. $\mathrm{Cu} K_{\alpha}$ large-angle diffraction intensity as a function of $2 \theta$ for $\mathrm{Dy} / \mathrm{Fe}$ samples.

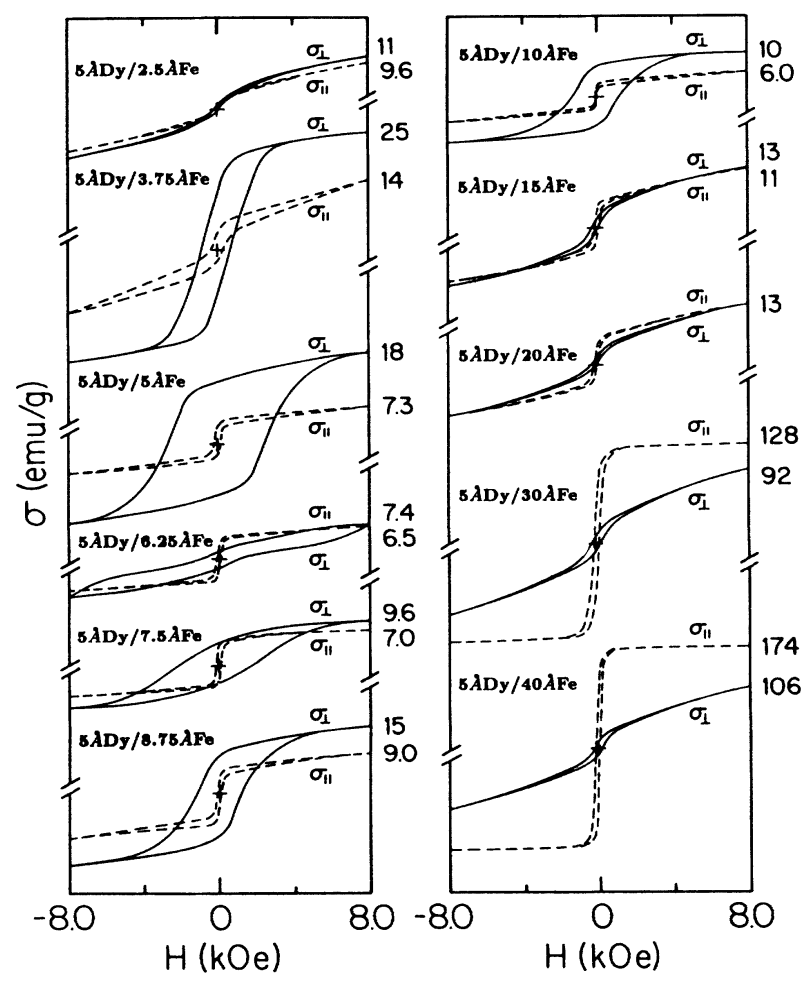

FIG. 15. Layer-thickness dependence of hysteresis loops for $(5 \AA \mathrm{Dy}) /(X \AA \mathrm{Fe})(X=2.5,3.75,5,6.25,7.5,8.75,10,15,20$, 30 , and 40$)$ at $300 \mathrm{~K}$.

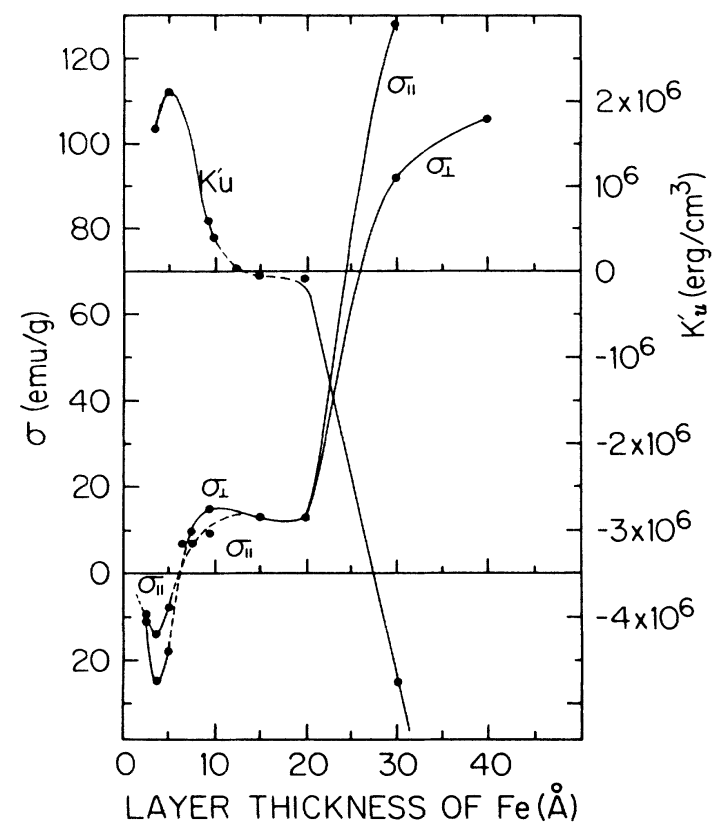

FIG. 16. Layer-thickness dependence of magnetization and measured anisotropy for ( $5 \AA \mathrm{Dy}) /(X \AA \mathrm{Fe})$ at $300 \mathrm{~K}$.

$\mathrm{Tb}) /(8.75 \AA \mathrm{Ae})$. A model calculation has been made for this sample in paper II]. Therefore the magnetization exhibits a "kink" there. (4) As $X>20 \AA$, the Fe has crystalline structure and its moment increases rapidly as shown in the Figs. 15 and 16 . (5) It is noticed that the four samples of $(5 \AA \mathrm{Dy}) /(X \AA \mathrm{Fe})(X=2.5,6.25,15,20)$ exhibit the character of smaller magnitude of magnetization and this can be interpreted as follows. Sample $(5 \AA$ Dy) $/(2.5 \AA \mathrm{Fe})$ is just ordered weakly by the exchange interactions between Dy and Fe moments and has almost homogeneous distribution of $\mathrm{Fe}$ and $\mathrm{Dy}$ constituents because both the $\mathrm{Fe}$ and Dy layers are very thin. Sample (5 $\AA \mathrm{Dy}) /(6.25 \AA \mathrm{Fe})$ displays almost zero magnetization since the Dy moment dominates in the Dy region and the $\mathrm{Fe}$ moment dominates in the Fe region and they compensate each other to produce a nearly zero net magnetization. However sample $(5 \AA \mathrm{Dy}) /(15 \AA \mathrm{Fe})$ [or $(5 \AA$ $\mathrm{Dy}) /(20 \AA \mathrm{Fe})]$ has much thicker Fe layer and the central $\mathrm{Fe}$ region gives no magnetization contribution as mentioned above. (6) As the Dy layer becomes thicker (not shown in Fig. 16) the compensation point will move toward larger Fe layer thickness and this is understandable from the antiferromagnetic coupling of $\mathrm{Dy}$ and $\mathrm{Fe}$ moments.

The layer-thickness dependence of the magnetic anisotropy, as shown in Figs. 15 and 16, exhibits the following features. (1) Figure 15 shows that the hysteresis loops of $\sigma_{\|}$change their shapes very little as the Fe layer thickness varies from 3.75 to $20 \AA$, whereas the hysteresis loops of $\sigma_{\perp}$ change their characteristics noticeably in the same range. When the Fe layer is thicker than $30 \AA$, the hysteresis loops of $\sigma_{\|}$also change noticeably. This implies that the magnetic properties in the direction perpendicular to the film plane strongly depend on the "inter- 
face" which is characterized by the anisotropic distribution of constituent atoms, but the magnetic properties in the parallel direction are mainly determined by the "inner part" of the Fe layer. (2) The samples with the layer thickness thinner than about $12 \AA$ usually have perpendicular anisotropy for both of the $2.5 \AA<X<6.25 \AA$ region where the Dy moment dominates and the 6.25 $\AA<X<12 \AA$ region where the $\mathrm{Fe}$ moment dominates. This suggests that the source of the perpendicular anisotropy is related to the anisotropic distribution and constituent magnetization rather than their net magnetization. (3) The behavior of $K_{u}$ at the compensation point is uncertain. Sato ${ }^{33,34}$ reported that $K_{u}=0$ at the compensation point for $\mathrm{Dy} / \mathrm{Fe}, \mathrm{Tb} / \mathrm{Fe}$, and $\mathrm{Gd} / \mathrm{Fe} \mathrm{CMF}$. But in the case of nominally homogeneous RE-TM films Van Dover $^{16}$ and Egami ${ }^{60}$ claimed that $K_{u}$ changes smoothly through the compensation point. This problem will be discussed in more detail in the following.

It is obvious that the torque magnetometer is unsuited to measure the torque curve in the vicinity of compensation point. However one might conclude that there is no anisotropy in terms of $M_{1}\left(H_{a}\right)$ and $M_{\|}\left(H_{a}\right)$ loops at the compensation point since

$$
K_{u}=K_{u}^{\prime} \pm 2 \pi M_{s}^{2}\left(+: M_{\perp}>M_{\|} ;-: M_{\perp}<M_{\|}\right) .
$$

At the compensation point $M_{\perp}\left(H_{a}\right)=M_{\|}\left(H_{a}\right)=0$ which leads to $K_{u}^{\prime}=0$, and subsequently $K_{u}=0$. But as point out in paper II, the origin of the anisotropy for $\mathrm{Dy} / \mathrm{Fe}$ CMF is the single-ion anisotropy of Dy ions which is proportional to the Dy magnetization squared, $M_{z}^{2}(\mathrm{Dy})$. The Dy magnetization changes smoothly through the compensation point, and thus it seems reasonable to conclude that the anisotropy changes smoothly through the compensation point. But this conclusion conflicts with the above one based on the formula of $K_{u}=K_{u}^{\prime} \pm 2 \pi M_{s}^{2}$. A possible interpretation is that the method based on Eq. (2) is unsuitable at the compensation point for $\mathrm{Dy} / \mathrm{Fe} \mathrm{CMF}$ which is characterized by the antiferromagnetically coupling between Dy and Fe subnetworks. A more detailed discussion is given in Appendix A of Ref. 46, and the conclusion is that the $K_{u}$ should change smoothly through the compensation point as shown in Fig. 16 by the solid line.

\section{Temperature dependence of magnetic properties}

Figure 17 is an example of the temperature dependence of the magnetic properties for $(Y \AA \mathrm{Dy}) /(6 \AA \mathrm{Fe})(Y=3$, 5,8 , and 14). The discussion analogous to what we have done for Dy/Co CMF in Fig. 10 is simplified as follows. (1) The magnetization at $4.2 \mathrm{~K}$ is much larger than those at $300 \mathrm{~K}$ since Dy is magnetically ordered at $4.2 \mathrm{~K}$. (2) At $4.2 \mathrm{~K}$ the samples possess a giant coercivity usually, e.g., $H_{c \perp}=59 \mathrm{kOe}$ for $(5 \AA \mathrm{Dy}) /(6 \AA \mathrm{Fe})$ and $H_{c \perp}=37$ kOe for $(14 \AA \mathrm{Dy}) /(6 \AA \mathrm{Fe})$ which is very weakly magnetically ordered at $300 \mathrm{~K}$. The coercivity $H_{c}$ of pure Dy film is about $10 \mathrm{kOe}$ at $4.2 \mathrm{~K}$. Therefore all the samples in Fig. 17 have a coercivity larger than that of pure Dy film at 4.2 K. (3) For all four samples, although $H_{c \perp}>H_{c \|}$ at $4.2 \mathrm{~K}$ the anisotropy value is difficult to mea-

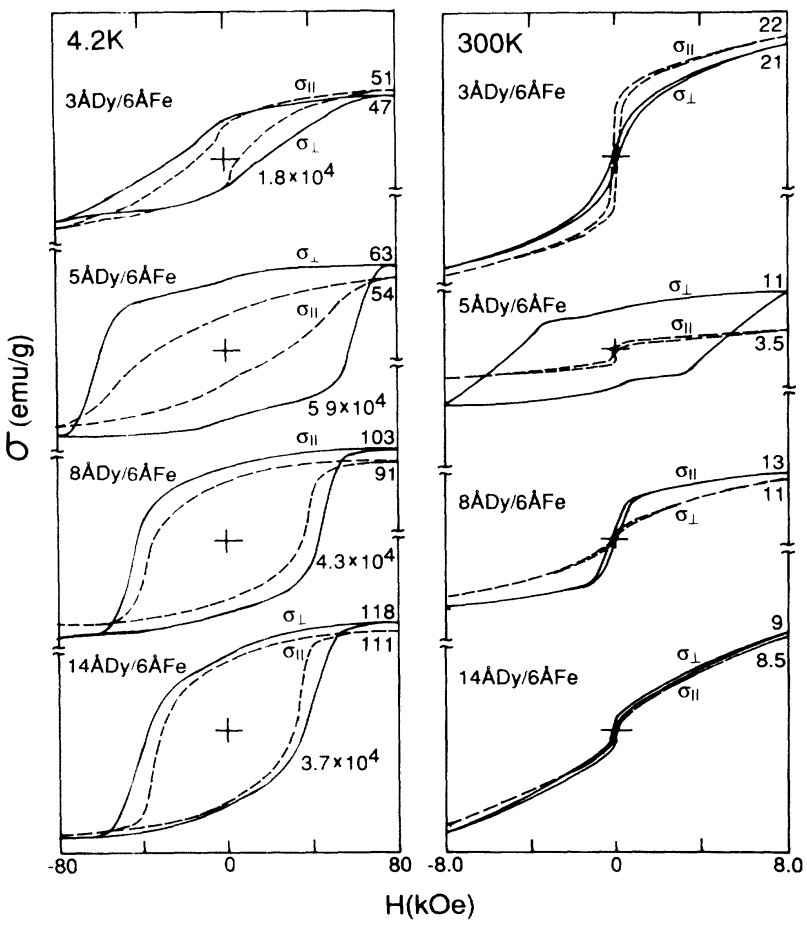

FIG. 17. Hysteresis loops for $(Y \AA \mathrm{Dy}) /(6 \AA \mathrm{Fe})(Y=3,5,8$, $14)$ at $300 \mathrm{~K}$ and $4.2 \mathrm{~K}$.

sure by the torque magnetometer because of the giant $H_{c}$ value and by the hysteresis loops because of the impossibility to define an area between $\sigma_{\perp}\left(H_{a}\right)$ and $\sigma_{\|}\left(H_{a}\right)$ curves.

The temperature dependence of the hysteresis loops for $(5 \AA \mathrm{Dy}) /(8 \AA \mathrm{Fe})$ is illustrated in Fig. 18(a) and their corresponding $\sigma$ and $H_{c}$ in Fig. 18(b). The following
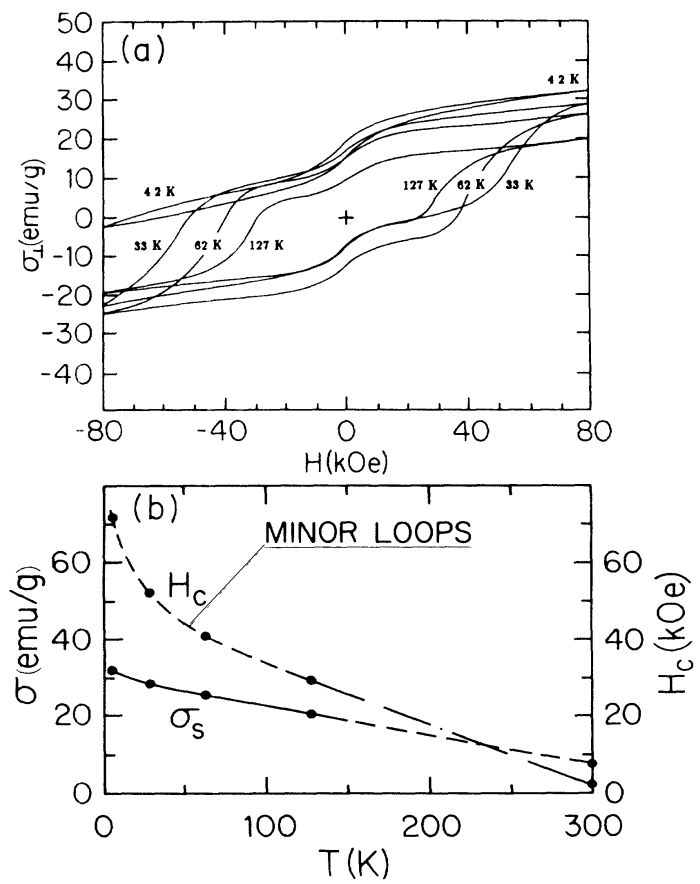

FIG. 18. Temperature dependence of hysteresis loops (a) and magnetization and coercivity for $(5 \AA \mathrm{Dy}) /(8 \AA \mathrm{Fe})(\mathrm{b})$. 
features should be noticed. (1) This sample shows large coercivity and constricted loops, and at $4.2 \mathrm{~K}$ exhibits a minor loop, which is an evidence of a system with both soft and hard magnetic phases. (2) Compared with Dy/Co CMF, Dy/Fe shows larger coercivity (see Fig. 12) and anisotropy (see Fig. 5 of paper II).

The temperature dependence of the torque curve for $(5$ $\AA \mathrm{Dy}) /(6 \AA \mathrm{Fe})$ has been performed. All three torque curves indicate that this sample has perpendicular anisotropy at 300,200 , and $77 \mathrm{~K}$. We notice that the torque curve is a nice sinusoidal at $77 \mathrm{~K}$ and has serious distortion at $300 \mathrm{~K}$ and $200 \mathrm{~K}$, particularly at $200 \mathrm{~K}$. The reason for this phenomenon is not yet fully clarified. It may be associated with the fact that the $200-\mathrm{K}$ sample ( 5 $\AA \mathrm{Dy}) /(6 \AA \mathrm{Fe})$ is close to the magnetic compensation point and the torque measurement is poorly suited to determine the magnetic property as was pointed out in a previous section.

\section{Discussion}

(a) Zwingman ${ }^{22}$ suggested that the perpendicular anisotropy appears to be induced by the stress from the substrate. We investigated the influence of substrate induced stress by producing a set of $(5 \AA \mathrm{Dy}) /(5 \AA \mathrm{Fe})$ samples on different substrates (Mylar, Ta, mica, and $\mathrm{Cu}$ ). It should be emphasized that the samples were made in one sputtering run to ensure the identical compositionally modulated structure and preparation condition for all four samples. This experiment proves that the stress induced anisotropy arising from the sample-substrate mismatch is not important based on the fact that all four hysteresis loops possess almost the same shape.

(b) The curves of $\lambda K_{u}^{\prime}$ vs Fe layer thickness $X$ are shown in Fig. 19. Several features are noticed. (1) All these curves have roughly the same intercept which corresponds to $K_{i}=2.5 \mathrm{erg} / \mathrm{cm}^{2}$. (2) The apparent volume anisotropy $K_{v}$ determined from the relation of slope $=\left(K_{v}-2 \pi M_{s}^{2}\right)$ has the order of magnitude of $10^{6}$ $\mathrm{erg} / \mathrm{cm}^{3}$. This value may have a considerable error due to the uncertainty in the $\boldsymbol{M}_{s}$ value. But the tendency of $K_{v}$ to increase as the Dy layer gets thicker is seen clearly. (3) The $K_{v}$ value determined from the slope is positive, which favors perpendicular anisotropy. But the slopes of all three curves are negative, i.e., $\left(K_{v}-2 \pi M_{s}^{2}\right)<0$, which means that the apparent volume anisotropy cannot compete with demagnetization anisotropy so that the interface anisotropy is the main source of perpendicular anisotropy.

\section{Dy/Ni CMF}

The magnetic properties of $\mathrm{Dy} / \mathrm{Ni} \mathrm{CMF}$ are significantly different from those of $\mathrm{Dy} / \mathrm{Co}$ and $\mathrm{Dy} / \mathrm{Fe}$. The Dy moment is disordered at room temperature. It is the exchange interactions between the TM and Dy atoms that give rise to the magnetic ordering of the Dy subnetwork. When the transition metal has necessary concentration and strong ferromagnetism, the exchange interactions may be powerful enough to raise the magnetic ordering temperature of Dy subnetwork above room tem-

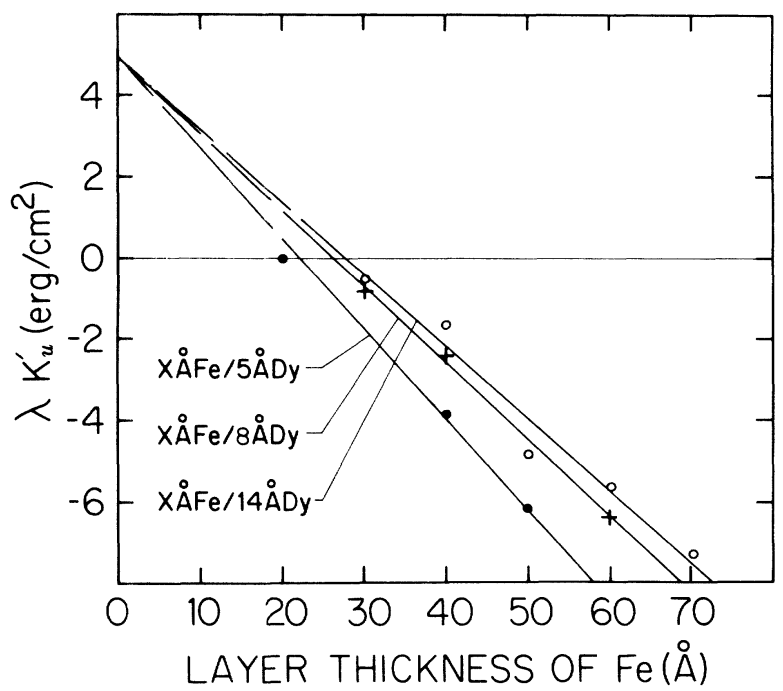

FIG. 19. The measured anisotropy $K_{u}^{\prime}$ multiplied by the bilayer thickness $\lambda$ vs the Fe layer thickness for the samples with the Dy layer thickness $Y=5,8,14 \AA$.

perature. We can expect that $\mathrm{Dy} / \mathrm{Ni} \mathrm{CMF}$ exhibits weaker magnetic properties because $\mathrm{Ni}$ has much weaker ferromagnetic characteristics than those of Co and $\mathrm{Fe}$.

We take the $(3.5 \AA \mathrm{Dy}) /(X \mathrm{~A} \mathrm{Ni})(X=5,10,15,20,30)$ as an example to discuss its magnetic properties briefly. First at room temperature, $(3.5 \AA \mathrm{Dy}) /(5 \AA \mathrm{Ni})$ is magnetically disordered, and the next two samples $(X=10$, 15) are only weakly ordered with the magnetization of $2 \sim 3 \mathrm{emu} / \mathrm{g}$ while the magnetization for $(3.5 \AA \mathrm{Dy}) /(10$ $\AA$ Co) reaches $103 \mathrm{emu} / \mathrm{g}$ (see Fig. 4). Second, that the perpendicular measured anisotropy (i.e., $K_{u}^{\prime}>0$ ) has not been observed yet in any of our $\mathrm{Dy} / \mathrm{Ni}$ samples at room temperature. Third, sample $(3.5 \AA \mathrm{Dy}) /(5 \AA \mathrm{Ni})$ and $(3.5$ $\AA \mathrm{Dy}) /(15 \AA \mathrm{Ni})$, which are magnetically disordered or very weakly ordered at room temperature, exhibit $K_{u}>0$ and strong ferromagnetism at $4.2 \mathrm{~K}$, but still $K_{u}^{\prime}<0$. In summary, Dy/Ni CMF shows much weaker magnetization and anisotropy characteristics compared with Dy/Co and Dy/Fe CMF.

\section{Tb/Fe CMF}

The magnetic properties of the $\mathrm{Tb}$ atom are similar to that of Dy: both have large atomic moments and singleion anisotropy, etc. Thus many similarities between $\mathrm{Dy} / \mathrm{Fe}$ and $\mathrm{Tb} / \mathrm{Fe} \mathrm{CMF}$ are expected. However some differences also will be pointed out in the following discussion.

\section{A. Layer-thickness dependence of magnetic properties}

An example of the $\mathrm{Fe}$ layer thickness dependence of hysteresis loops for $(4.5 \AA \mathrm{Tb}) /(X \AA \mathrm{Fe})$ is illustrated in Fig. 20 and the corresponding Fe layer-thickness dependences of magnetic parameters, such as magnetization and anisotropy, are given in Fig. 21. The similarities between Figs. 15 and 16 for $(5 \AA \mathrm{Dy}) /(X \AA \mathrm{Fe})$ and Figs. 20 and 21 for $(4.5 \AA \mathrm{Tb}) /(X \AA \mathrm{Fe})$ are obvious. For exam- 


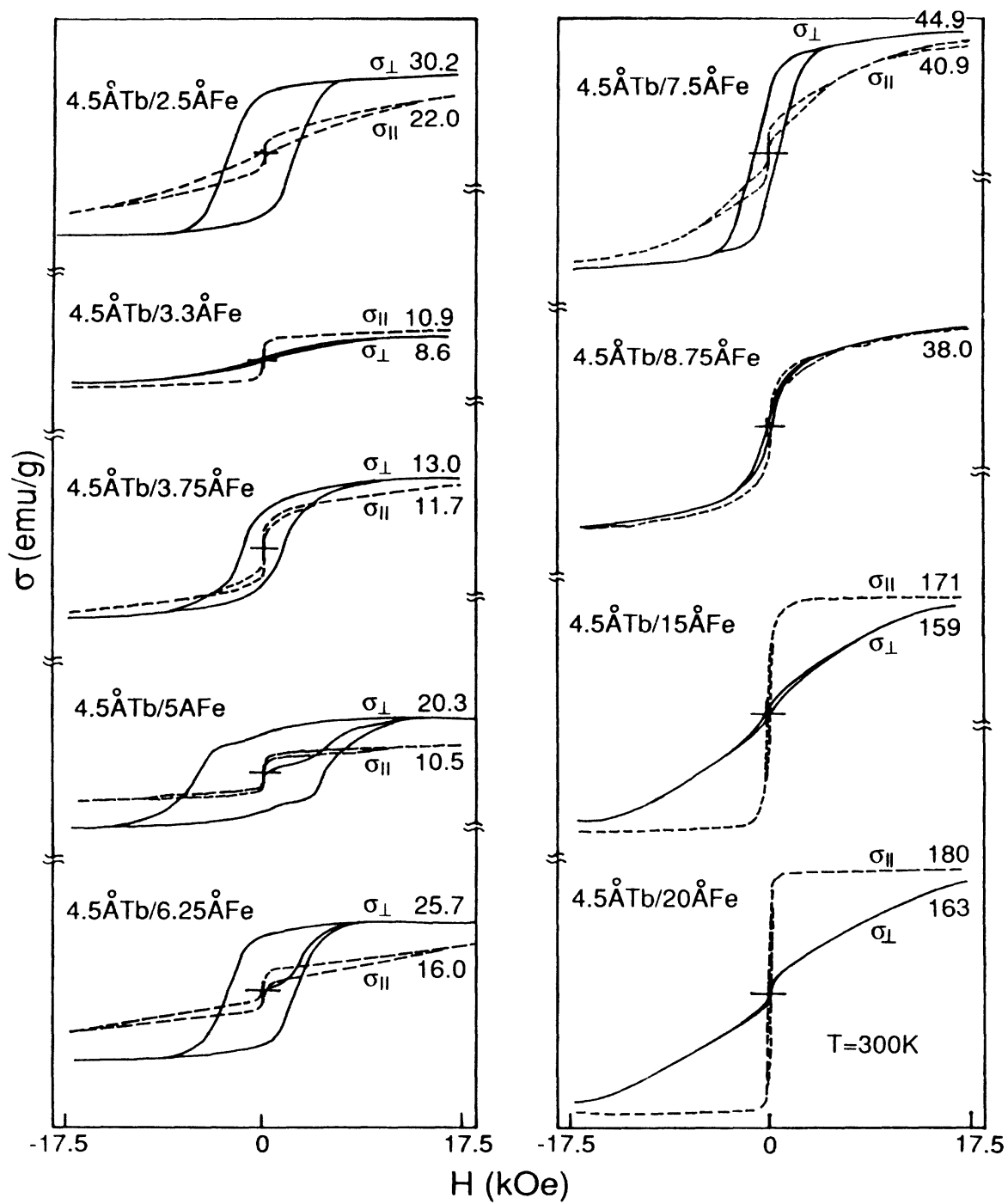

FIG. 20. Hysteresis loops for $(4.5 \AA \mathrm{Tb}) /(X \AA \mathrm{Ae})(X=2.5,3.3,3.75,5,6.25,7.5,8.75,15,20)$ at $300 \mathrm{~K}$.

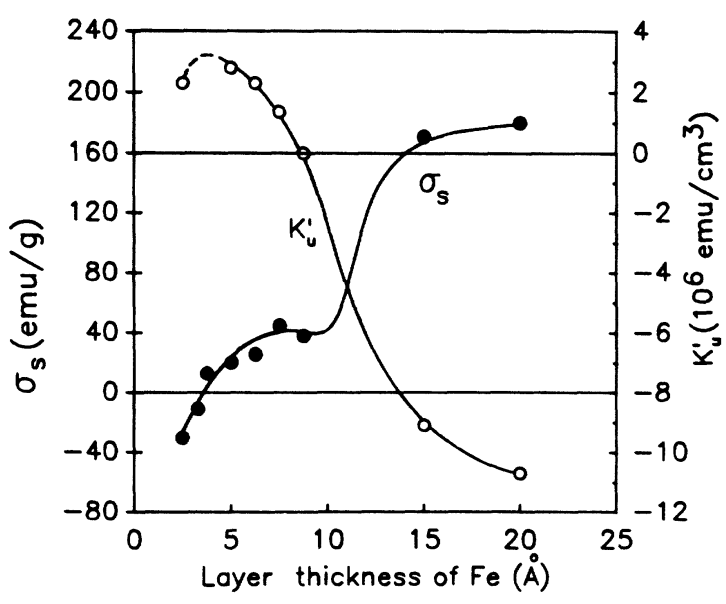

FIG. 21. Layer-thickness dependence of $\sigma_{s}$ and $K_{u}^{\prime}$ for $(4.5 \AA$ $\mathrm{Tb}) /(X \AA \mathrm{Fe})$ at $300 \mathrm{~K}$. ple, sample $(4.5 \AA \mathrm{Tb}) /(3.3 \AA \mathrm{Fe})$ is close to the compensation point at room temperature, and there is a magnetization "kink" around $X=10 \AA$. We skip the similar discussion for the present $\mathrm{Tb} / \mathrm{Fe} \mathrm{CMF}$ here. Compared with $\mathrm{Dy} / \mathrm{Fe} \mathrm{CMF}, \mathrm{Tb} / \mathrm{Fe} \mathrm{CMF}$ usually exhibits stronger perpendicular anisotropy and one example is illustrated in Fig. 5 of paper II.

\section{B. Temperature dependence of magnetic properties}

The temperature dependence of the hysteresis loops are presented in Figs. 20, 22, and 23. It is found (1) at $4.2 \mathrm{~K}$ the samples with $X<6.25 \AA$ exhibits giant coercivity. Sample $(4.5 \AA \mathrm{Tb}) /(2.5 \AA \mathrm{Fe})$ has $H_{c}=54 \mathrm{kOe}$, and samples $(4.5 \AA \mathrm{Tb}) /(X \AA \mathrm{Fe})(X=3.3,5,6.25)$ show minor hysteresis loops. (2) As the temperature decreases from $300 \mathrm{~K}$ to $4.2 \mathrm{~K}$, the change in shape of the hysteresis loops of thin $\mathrm{Fe}$ layer samples, e.g., $(4.5 \AA \mathrm{Tb}) /(X \AA \mathrm{Ae})$ $(X=2.5,3.3$, etc. $)$ is notable since the Tb moment becomes ordered at lower temperature. For the thick $\mathrm{Fe}$ 


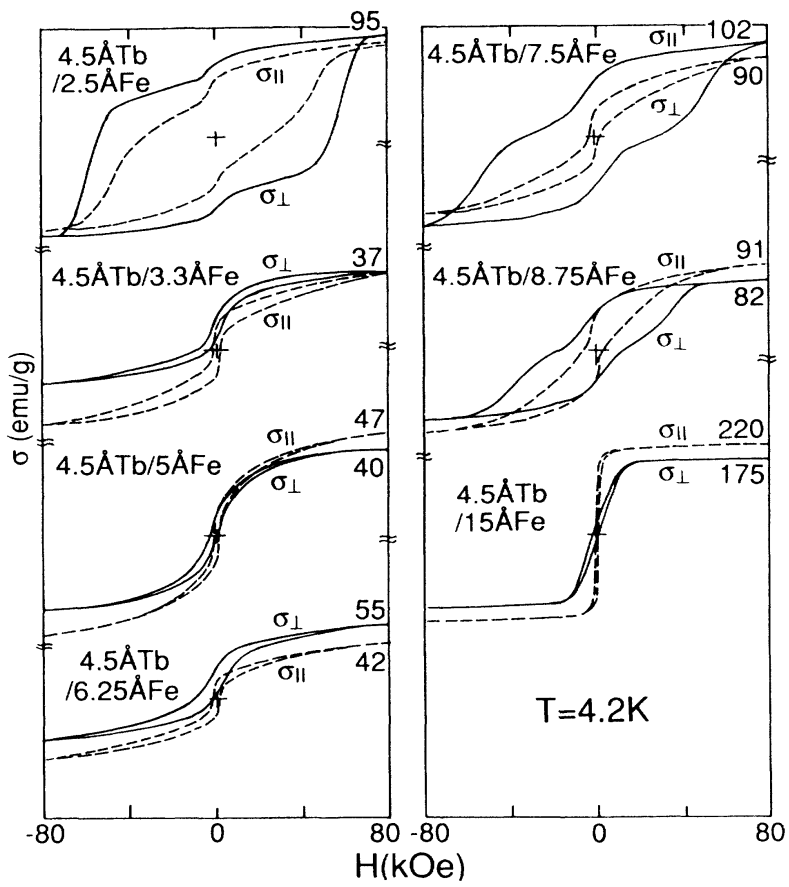

FIG. 22. Hysteresis loops for $(4.5 \AA \mathrm{Tb}) /(X \AA \mathrm{Fe})(X=2.5$, $3.3,5,6.25,7.5,8.75$, and 15$)$ at $4.2 \mathrm{~K}$.

layer samples, where Fe moment dominates, e.g., (4.5 ̊ $\mathrm{Tb}) /(15 \AA \mathrm{Fe})$, this change is much weaker. (3) Sample $(4.5 \AA \mathrm{Tb}) /(3.3 \AA \mathrm{Fe})$ exhibits a noteworthy temperature dependence of hysteresis loop, as shown in Fig. 23. The

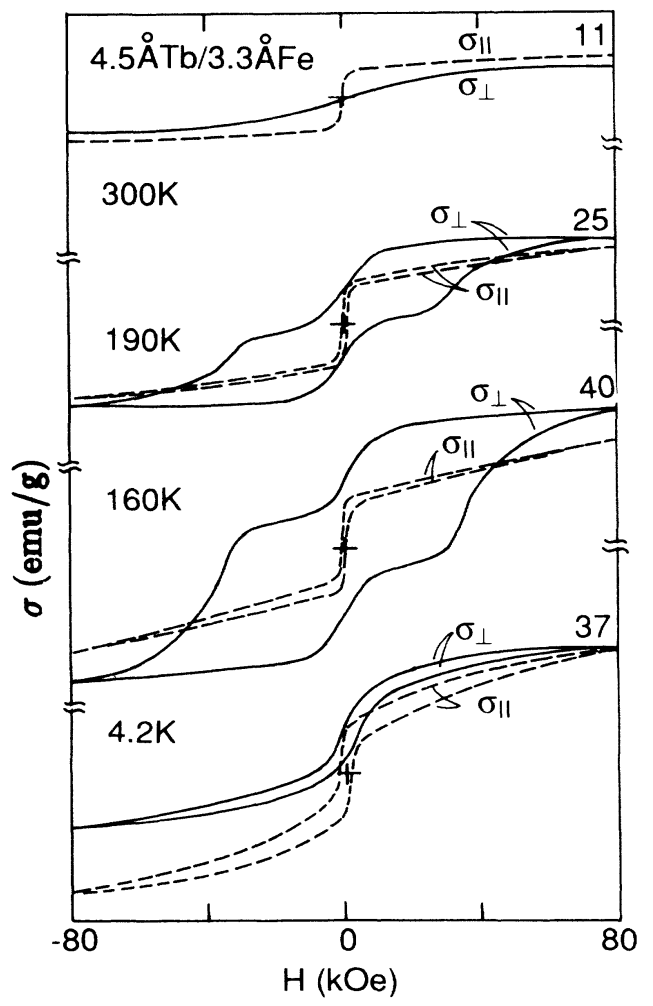

FIG. 23. Temperature dependence of hysteresis loops for $(4.5$ $\AA \mathrm{Tb}) /(3.3 \AA \mathrm{Fe})(X=300,190,160,4.2 \mathrm{~K})$. constricted hysteresis loops at $T<190 \mathrm{~K}$ suggest a twophase system. Phase 1 is the Tb-rich phase with hard magnetic properties which is spread over most of the sample, and phase 2 is the Fe-rich phase with soft magnetic properties in the central part of $\mathrm{Fe}$ region, whose moment can be flipped as the applied field is reversed. The resultant hysteresis loop is the sum of the two subhysteresis loops and has a constricted shape as expected. This feature is also observed for $(4.5 \AA \mathrm{Tb}) /(X \AA \mathrm{Fe})$ $(X=5,6.25,7.5,8.75)$ at $4.2 \mathrm{~K}$. For thicker Fe layer samples, e.g., $(4.5 \AA \mathrm{Tb}) /(15 \AA \mathrm{Fe})$ this feature disappears because the $\mathrm{Fe}$ moment strongly dominates. (4) Compared with $\mathrm{Dy} / \mathrm{Fe} \mathrm{CMF}, \mathrm{Tb} / \mathrm{Fe} \mathrm{CMF}$ demonstrates large coercivity in a wider range of $\mathrm{Fe}$ layer thickness as seen in Fig. 22. This may be due to the fact that $\mathrm{Tb} / \mathrm{Fe} \mathrm{CMF}$ possesses larger anisotropy or that the magnetic moment of $\mathrm{Tb}$ ion is coupled more strongly with the crystal field through the spin-orbit interaction.

\section{SUMMARY AND CONCLUSIONS}

The major conclusions from the experimental studies on Dy- and Tb-based CMF's are as follows. (1) Interesting magnetic behavior, such as large PMA and coercivity, is associated with the interfaces, which are characterized by the anisotropic distributions of the constituent atoms; only the CMF with nanoscale layer thicknesses show these notable magnetic properties. (2) The singleion anisotropy of RE ions possessing orbital angular momentum is the major source of PMA and this point will be discussed in detail in paper II. (3) The antiferromagnetic coupling between RE and TM moments is confirmed by the experimental measurements of the total magnetization depending on the layer thickness and temperature; this also will be proved by the modeling analyses of the distributions of the constituent magnetization along the film normal in paper II. (4) The intrinsic anisotropy $K_{u}$ and the constituent magnetizations change their values smoothly through the compensation point, and the coercivity divergence at the compensation point, as was reported for heavy $A^{\mathrm{RE}} / B^{\mathrm{TM}}$ homogeneous alloys, does not occur for the CMF structure. It should be emphasized that the magnetic properties of these CMF's are the results of the statistical average over the whole sample, and in paper II we will derive some micromagnetic information for the CMF structure on the basis of a mean-field model along with a single-ion anisotropy model.

\section{ACKNOWLEDGMENTS}

We would like to acknowledge the valuable discussions with S. S. Jaswal, R. D. Kirby, and K. D. Aylesworth. This work was supported by National Science Foundation (NSF) under Grant Nos. DMR-86-05367 and INT87-15441. 
*Permanent address: Department of Electronic Engineering, Hangzhou University, Hangzhou, Zhejiang, People's Republic of China.

${ }^{1}$ P. Chaudhari, J. J. Cuomo, and R. J. Gambino, IBM J. Rev. 17, 66 (1973).

${ }^{2}$ R. C. Taylor, J. Appl. Phys. 47, 1164 (1976).

${ }^{3}$ R. C. Taylor and A. Gangulee, J. Appl. Phys. 47, 4666 (1976).

${ }^{4}$ T. Katayama, M. Hirano, Y. Koizumi, K. Kawanishi, and T. Tsushima, IEEE Trans. Magn. MAG-13, 1603 (1977).

${ }^{5}$ H. Kobayashi, T. Ono, A. Tsushima, and T. Suzuki, Appl. Phys. Lett. 43, 389 (1983).

${ }^{6}$ K. Tsutsumi and H. Sugahara, Jpn. J. Appl. Phys. Pt. 2 23, L169 (1984).

${ }^{7}$ M. Hong, E. M. Gyorgy, and D. D. Bacon, Appl. Phys. Lett. 44, 706 (1984).

${ }^{8}$ H. K. Lachowicz, IEEE Trans. Magn. MAG-20, 1417 (1984).

${ }^{9}$ H. A. Algra, K. H. J. Buschow, and R. A. Henskens, J. Phys. Colloq. 41, C8-646 (1980).

${ }^{10}$ R. Carey, P. A. O'Rourke, and B. W. Thomas, J. Magn. Magn. Mater. 50, 335 (1985).

${ }^{11}$ T. Suzuki, Jpn. J. Appl. Phys. Pt. 2 24, L199 (1985).

${ }^{12}$ T. Suzuki, J. Magn. Magn. Mater. 54-57, 1407 (1986).

${ }^{13}$ Y. Mimura, N. Imamura, T. Kobayshi, A. Okada, and Y. Kushiro, J. Appl. Phys. 49, 1208 (1978).

${ }^{14}$ R. Sato, N. Sato, and Y. Togami, Jpn. J. Appl. Phys. Pt. 2 24, L266 (1985).

${ }^{15}$ T. Niihara, S. Takayama, and Y. Sugita, IEEE Trans. Magn. MAG-21, 1638 (1985).

${ }^{16}$ R. B. Van Dover, M. Hong, E. M. Gyorgy, J. F. Dillon, Jr., and S. D. Albiston, J. Appl. Phys. 57, 3897 (1985).

${ }^{17}$ R. Krishnan, M. Porte, M. Tessier, J. P. Vitton, and Y. Le Cars, IEEE Trans. Magn. MAG-24, 1773 (1988).

${ }^{18}$ R. W. Cochrane, R. Harris, and M. J. Zuckermann, Phys. Rep. 48, 1 (1978).

${ }^{19}$ R. J. Gambino, P. Chaudhari, and J. J. Cuomo, in Magnetism and Magnetic Materials-1973 (Boston), Proceedings of the 19th Annual Conference on Magnetism and Magnetic Materials, AIP Conf. Proc. No. 18, edited by C. D. Graham and J. J. Rhyne (AIP, New York, 1974), p. 578.

${ }^{20}$ N. Heiman, A. Onton, D. F. Kyser, Kenneth Lee, and C. R. Guarneri, in Magnetism and Magnetic Materials-1974 (San Francisco), Proceedings of the 20th Annual Conference on Magnetism and Magnetic Materials, AIP Conf. Proc. No. 24, edited by C. D. Graham, G. H. Lander, and J. J. Rhyne (AIP, New York, 1975), p. 573.

${ }^{21} \mathrm{~S}$. Esho, and S. Fujiwara, in Magnetism and Magnetic Materials-1976 (Joint NMM-Intermag, Conference, Pittsburgh), AIP Conf. Proc. No. 34, edited by J. J. Becker and G. H. Lander (AIP, New York, 1976), p. 331.

${ }^{22}$ Zwingman, W. L. Wilson, Jr., and H. C. Bourne, Jr., in Magnetism and Magnetic Materials-1976 (Ref. 21), p. 334.

${ }^{23} \mathrm{~K}$. Twarowski, H. K. Lachowicz, M. Gutowski, and $\mathbf{H}$. Szymczk, Phys. Status Solidi A 63, 103 (1981).

${ }^{24}$ W. H. Meiklejohn, F. E. Luborsky, and P. G. Frischman, IEEE Trans. Magn. MAG-23, 2272 (1987).

${ }^{25}$ G. S. Cargill III and T. Mizoguchi, J. Appl. Phys. 49, 1753 (1978).

${ }^{26}$ T. Mizoguchi and G. S. Cargill III, J. Appl. Phys. 50, 3570 (1979).

${ }^{27}$ Y. Suzuki, S. Takyma, F. Kirino, and N. Ohta, IEEE Trans. Magn. MAG-23, 2275 (1987).

${ }^{28}$ Y. Suzuki and N. Ohta, J. Appl. Phys. 63, 3633 (1988).

${ }^{29}$ A. Yoshihara, M. Takahashi, T. Shimamori, T. Wakiyama,
M. Miyazaki, S. Yamaguchi, and K. Hayashi, J. Appl. Phys. 49, 5492 (1978).

${ }^{30}$ T. Miyazaki, K. Hayashi, S. Yamaguchi, M. Takahashi, A. Yoshihara, T. Shimaori, and T. Wakiyama, J. Magn. Magn. Mater. 75, 243 (1988).

${ }^{31}$ M. Takahashi, A. Yoshiara, T. Shimamori, T. Wakiyama, T. Miyazaki, K. Hayashi, and S. Yamaguchi, J. Magn. Magn. Mater. 75, 252 (1988).

${ }^{32}$ K. H. J. Buschow, J. Appl. Phys. 63, 3130 (1988).

${ }^{33}$ N. Sato, J. Appl. Phys. 59, 2514 (1986).

${ }^{34}$ N. Sato and K. Habu, J. Appl. Phys. 61, 4287 (1987).

${ }^{35}$ K. Yamauchi, K. Habu, and N. Sato, J. Appl. Phys. 64, 5478 (1988).

${ }^{36}$ N. Sato, K. Habu, and T. Oyama, IEEE Trans. Magn. MAG23, 2614 (1987).

${ }^{37}$ S. Honda, M. Nawate, M. Yoshiyama, and T. Kuda, IEEE Trans. Magn. MAG-23, 2952 (1987).

${ }^{38}$ S. C. Shin, M. L. Cofied, and R. H. D. Nuttall, J. Appl. Phys. 61, 4326 (1987).

${ }^{39}$ Y. Suzuki, H. Masuda, T. Shibata, and N. Koshizuka, IEEE Trans. Magn. MAG-23, 3704 (1987).

${ }^{40}$ D. J. Sellmyer, Z. R. Zhao, Z. S. Shan, and S. Nafis, J. Appl. Phys. 61, 4323 (1987).

${ }^{41}$ Z. S. Shan, S. Nafis, K. D. Ayleworth, and D. J. Sellmyer, J. Appl. Phys. 63, 3218 (1988).

${ }^{42}$ Z. S. Shan and D. J. Sellmyer, J. Appl. Phys. 64, 5745 (1988).

${ }^{43}$ D. J. Sellmyer and Z. S. Shan, in Proceedings of the Materials Research Society International Meeting on Advanced Materials, Multilayers Symposium, Tokyo, 1988. (Materials Research Society, Pittsburgh, 1989), Vol. 10, p. 269.

${ }^{44}$ D. J. Sellmyer, J. A. Woollam, Z. S. Shan, and W. A. McGahan, Proceedings of the Materials Research Society Meeting on Magneto-Optic Data Storage, San Diego, 1989 (Materials Research Society, Pittsburgh, 1990) edited by C. J. Robinson et al., Vol. 150, p. 51.

${ }^{45}$ Z. S. Shan, D. J. Sellmyer, S. S. Jaswal, Y. J. Wang, and J. X. Shen, Phys. Rev. Lett. 63, 449 (1989).

${ }^{46}$ Z. S. Shan, Ph.D. dissertation, University of Nebraska, 1990.

${ }^{47}$ P. F. Carcia, A. D. Meinhaldt, and A. Suna, Appl. Phys. Lett. 47, 178 (1985).

${ }^{48}$ P. F. Carcia, J. Vac. Sci. Technol. A5, 1975 (1987).

${ }^{49}$ P. F. Carcia, J. Appl. Phys. 63, 5066 (1988).

${ }^{50}$ F. J. A. den Broeder, H. C. Donkersloot, H. J. G. Draaisma, and W. J. M. de Jonge, J. Appl. Phys. 61, 4317 (1987).

${ }^{51}$ H. J. G. Draaisma, W. J. M. de Jonge, and F. J. A den Broder, J. Magn. Magn. Mater. 66, 351 (1987).

${ }^{52}$ F. J. A. den Broeder, D. Kuiper, and H. J. G. Draaisma, IEEE Trans. Magn. MAG-23, 3696 (1987).

${ }^{53}$ H. J. G. Draaisma, F. J. A. den Broeder, and W. J. M. de Jonge, J. Appl. Phys. 63, 3479 (1988).

${ }^{54}$ H. J. G. Draaisma and W. J. M. de Jonge, J. Appl. Phys. 62, 3318 (1987).

${ }^{55}$ F. J. A. den Broeder, D. Kuiper, A. P. Van de Mosselaer, and W. Hoving, Phys. Rev. Lett. 60, 2769 (1988).

${ }^{56}$ N. C. Koon, B. T. Jonker, F. A. Volkening, J. J. Krebs, and G. A. Prinz, Phys. Rev. Lett. 59, 2463 (1987).

${ }^{57}$ M. L. Neel, J. Phys. Radium 15, 225 (1954).

${ }^{58}$ C. G. Robbins (unpublished).

${ }^{59}$ J. J. Rhyne, IEEE Trans. Magn. MAG-21, 1990 (1985).

${ }^{60}$ T. Egami, C. D. Graham, Jr., W. Dmowksi, P. Zhou, P. J. Flanders, E. E. Marinero, H. Notary, and C. Robison, IEEE Trans. Magn. MAG-23, 2269 (1987). 